\title{
A functional deficiency of TERA/VCP/p97 contributes to impaired DNA repair in multiple polyglutamine diseases
}

Kyota Fujita ${ }^{1, \star}$, Yoko Nakamura ${ }^{1, \star}$, Tsutomu Oka ${ }^{1, \star}$, Hikaru Ito ${ }^{1}$, Takuya Tamura ${ }^{1}$, Kazuhiko Tagawa1, Toshikazu Sasabe ${ }^{1}$, Asuka Katsuta1,2, Kazumi Motoki ${ }^{1}$, Hiroki Shiwaku1 ${ }^{1}$, Masaki Sone ${ }^{1,2}$, Chisato Yoshida1, Masahisa Katsuno ${ }^{3}$, Yoshinobu Eishi ${ }^{4}$, Miho Murata ${ }^{5}$, J. Paul Taylor ${ }^{6}$, Erich E. Wanker ${ }^{7}$, Kazuteru Kono ${ }^{8}$, Satoshi Tashiro ${ }^{8}$, Gen Sobue ${ }^{3}$, Albert R. La Spada ${ }^{9}$ \& Hitoshi Okazawa ${ }^{1}$

It is hypothesized that a common underlying mechanism links multiple neurodegenerative disorders. Here we show that transitional endoplasmic reticulum ATPase (TERA)/ valosin-containing protein (VCP)/p97 directly binds to multiple polyglutamine disease proteins (huntingtin, ataxin-1, ataxin-7 and androgen receptor) via polyglutamine sequence. Although normal and mutant polyglutamine proteins interact with TERA/VCP/p97, only mutant proteins affect dynamism of TERA/VCP/p97. Among multiple functions of TERA/VCP/p97, we reveal that functional defect of TERA/VCP/p97 in DNA double-stranded break repair is critical for the pathology of neurons in which TERA/VCP/p97 is located dominantly in the nucleus in vivo. Mutant polyglutamine proteins impair accumulation of TERA/VCP/p97 and interaction of related double-stranded break repair proteins, finally causing the increase of unrepaired double-stranded break. Consistently, the recovery of lifespan in polyglutamine disease fly models by TERA/VCP/p97 corresponds well to the improvement of double-stranded break in neurons. Taken together, our results provide a novel common pathomechanism in multiple polyglutamine diseases that is mediated by DNA repair function of TERA/VCP/p97.

\footnotetext{
${ }^{1}$ Department of Neuropathology, Medical Research Institute, Tokyo Medical and Dental University, 1-5-45, Yushima, Bunkyo-ku, Tokyo 113-8510, Japan. 2 Department of Biomolecular Science, Faculty of Science, Toho University, 2-2-1 Miyama, Funabashi 274-8510, Japan. ${ }^{3}$ Department of Neurology, Graduate School of Medicine, Nagoya University, 65, Tsurumai-cho, Showa-ku, Nagoya 466-8550, Japan. ${ }^{4}$ Department of Human Pathology, Graduate School of Medicine, Tokyo Medical and Dental University, 1-5-45, Yushima, Bunkyo-ku, Tokyo 113-8510, Japan. ${ }^{5}$ Department of Neurology, National Center of Neurology and Psychiatry, 4-1-1, Ogawahigashimachi, Kodaira 187-8551, Japan. ${ }^{6}$ Department of Developmental Neurobiology, St. Jude Children's Research Hospital, 262 Danny Thomas Place, Memphis, Tennessee 38105, USA. ${ }^{7}$ Department of Neurogenetics, Max-Delbrück Center for Molecular Medicine, 13125 Berlin-Buch, Germany. ${ }^{8}$ Department of Cellular Biology, Research Institute for Radiation Biology and Medicine, Hiroshima University, 1-2-3, Kasumi, Minami-ku, Hiroshima 734-8553, Japan. ${ }^{9}$ Departments of Pediatrics, Cellular and Molecular Medicine, and Neurosciences, Division of Biological Sciences, and the Institute for Genomic Medicine, University of California, San Diego, La Jolla, California 92093, USA. * These authors contributed equally to this work. Correspondence and requests for materials should be addressed to H.O. (email: okazawa-tky@umin.ac.jp).
} 
$\mathrm{T}$ ransitional endoplasmic reticulum (ER) ATPase (TERA), also known as valosin-containing protein (VCP) or p97, is implicated in various neurodegenerative disorders. Mutations in the human TERA/VCP/p97 gene cause frontotemporal dementia associated with inclusion body myopathy and earlyonset Paget disease $^{1}$ or familial amyotrophic lateral sclerosis ${ }^{2}$, and $T E R A / V C P / p 97$ is now considered a major causative gene for frontotemporal lobar degeneration (FTLD). The TERA/VCP/p97 protein also interacts with a normal length polyglutamine (polyQ) tract sequence derived from a transcription factor, Brn2 (ref. 3), and the mutant form of a polyglutamine disease protein, ataxin-3 (ref. 4), which causes spinocerebellar ataxia type 3.

TERA/VCP/p97 is a member of the ATPase associated with various activities $\mathrm{AAA}+$ protein family involved in diverse cellular functions. The name TERA is derived from its function in the membrane transfer from the ER to the Golgi apparatus. TERA/VCP/p97 contains ATPase catalytic domains and regulatory N-terminal and C-terminal domains; it forms a hexameric structure and couples ATP hydrolysis to cellular activities ${ }^{5}$. For instance, the budding process of transition vesicles from smooth and rough ER is ATP-dependent and requires TERA/VCP/p97 (ref. 6). TERA/VCP/p97 is essential for cistern reformation from mitotic Golgi fragments ${ }^{7}$. TERA/VCP/p97 also interacts with clathrin $^{8}$, which coats the endosome membrane.

TERA/VCP/p97 has a critical role in ER-associated protein degradation (ERAD) though interactions with Derlin-1, VCPinteracting membrane protein ${ }^{9}$ and UFD1/NPL4 complex ${ }^{10}$. TERA/VCP/p97 supplies energy to this protein complex to extract misfolded proteins from the ER lumen and transport them to the proteasome for degradation. TERA/VCP/p97 may directly interact with gp78, a sensor of misfolded proteins, on the ER membrane ${ }^{11}$, and may also interact with small interfering RNA to affect cell division ${ }^{12}$. Furthermore, a recent report revealed a role of TERA/VCP/p97 in DNA double-stranded break (DSB) repair, as TERA/VCP/p97 interacts with and promotes release of lethal (3) malignant brain tumor-like protein 1 (L3MBTL1) from DSBs and recruitment of p53-binding protein-1 (53BP1) ${ }^{13,14}$. Hence, TERA/VCP/p97 regulates ERAD, membrane transport and DSB repair.

Despite these findings, it remains unclear how TERA/VCP/p97 is altered in FTLD or motor neuron disease associated with TERA/VCP/p97 gene mutations. RNAi-mediated depletion of $T E R A / V C P / p 97$ induces accumulation of ubiquitinated proteins, vacuolization of ER, impairment of protein trafficking through the secretory pathway, and induces apoptosis in cell lines ${ }^{15-17}$. However, overexpression of mutant TERA/VCP/p97 causes degeneration phenotypes in vitro ${ }^{18}$ and in vivo ${ }^{19-21}$. ATPase activity was increased in some experiments using the GST-TERA/ $\mathrm{VCP} / \mathrm{p} 97$ fusion protein ${ }^{22,23}$. In spinocerebellar ataxia type 3 , mutant ataxin-3 changes TERA/VCP/p97 modification, promotes nuclear translocation of TERA/VCP/p97, and suppresses transcription ${ }^{24}$, suggesting a pathological role of TERA/VCP/ p97 in the nucleus. Like FTLD, these results have not yet been verified, and it is unclear how ATPase or ERAD activities and the other functions of TERA/VCP/p97 are affected in neurodegeneration.

Our previous observation that TERA/VCP/p97 physically interacts with the polyQ sequence ${ }^{3}$ prompted us to revisit the question whether TERA/VCP/p97 has a common pathological role in multiple polyQ diseases. As expected, we show that multiple polyQ disease proteins for bulbospinal muscular atrophy/Kennedy's disease, spinocerebellar ataxia type 1 (SCA1), spinocerebellar ataxia type 7, and Huntington's disease (HD) physically interact with TERA/VCP/p97. Though TERA/ $\mathrm{VCP} / \mathrm{p} 97$ binds to normal as well as mutant polyQ proteins, mutant polyQ proteins specifically affect dynamism of TERA/
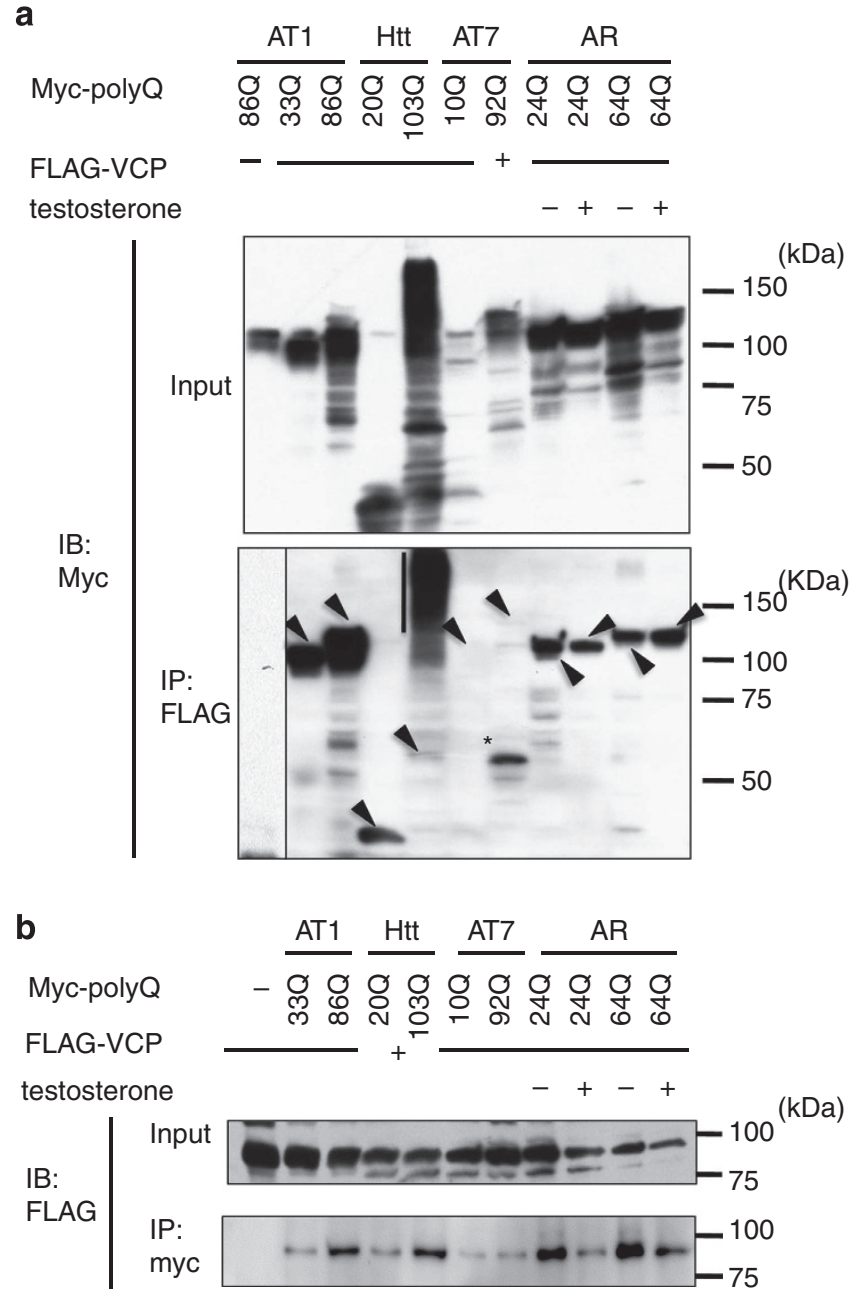

Figure 1 | Multiple polyQ proteins interact with TERA/VCP/p97. (a) VCP coprecipitates with normal as well as mutant forms of ataxin-1 (ATXN1), huntingtin (Htt), full-length ataxin-7 (ATXN7) or androgen receptor (AR). HeLa cells were co-transfected with Myc-polyQ and FLAG-VCP protein expression vectors, and the cell lysates were immunoprecipitated with antiFLAG antibody. Lane 1 is a negative control in which HeLa cells are transfected with Myc-ATXN1-86Q expression vector only. Coprecipitation of ATXN7 is relatively unstable. AR binds strongly to $\mathrm{VCP}$ in the absence of testosterone $(T-)$ but only weakly in the presence of testosterone $(T+)$ because of the dissociation of their intracellular distribution. Arrows indicate co-precipitated full-length proteins, and bar indicates co-precipitated Htt aggregates.

(b) Normal and mutant polyQ proteins coprecipitate with VCP. ATXN7 did not obviously coprecipitate in some cases. Mutant Htt seems to bind more strongly to VCP than to normal Htt in these blots; however, the difference is not definite in other blots.

VCP/p97 and impair its function. Mislocalization of TERA/VCP/ p97 to aggregates is confirmed in diseased human brains.

More surprisingly, TERA/VCP/p97 is localized to the nucleus in human and mouse adult neuron in vivo. Live imaging reveals that mutant polyQ proteins specifically impair recruitment of TERA/VCP/p97 to DNA damage foci and inhibit DSB repair. Suppression of ERAD is relatively weak in primary neurons from mutant ATXN1-knock in (KI) mice. TERA/VCP/p97 genetically rescues phenotypes in Drosophila HD and SCA1 models in consistence with the recovery of DSB. Taken together, we propose a novel hypothesis that impairment of TERA/VCP/p97-mediated DSB repair is a common pathology in multiple polyQ diseases. 
a
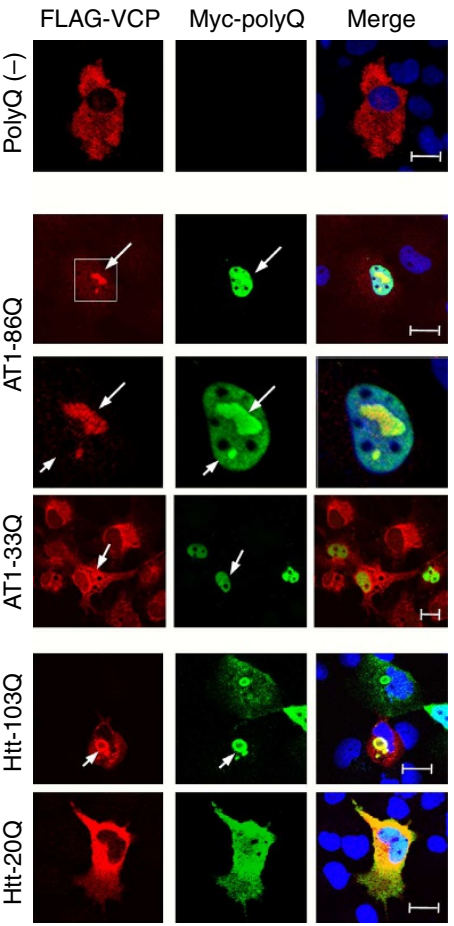

b

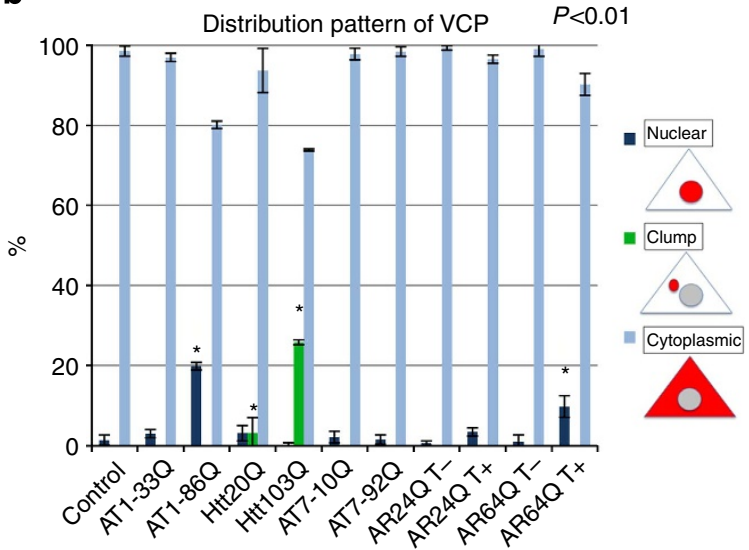

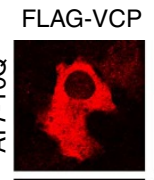
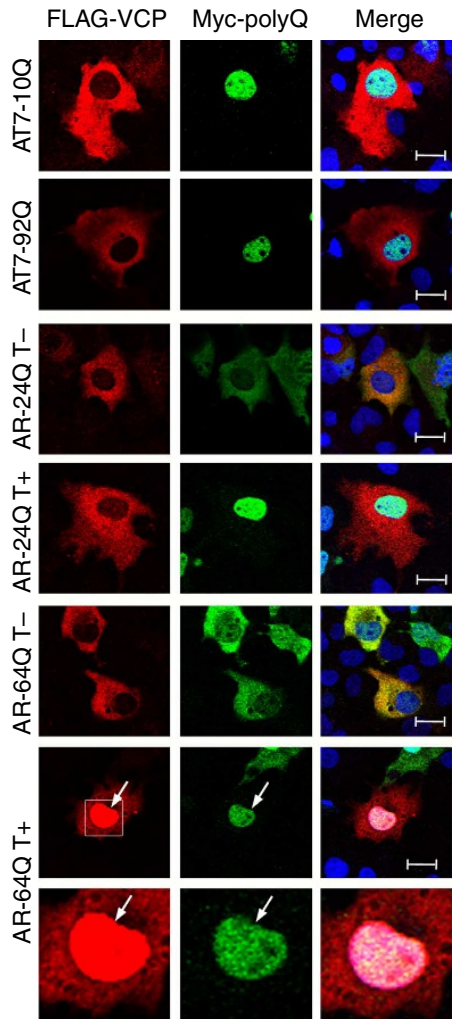

C
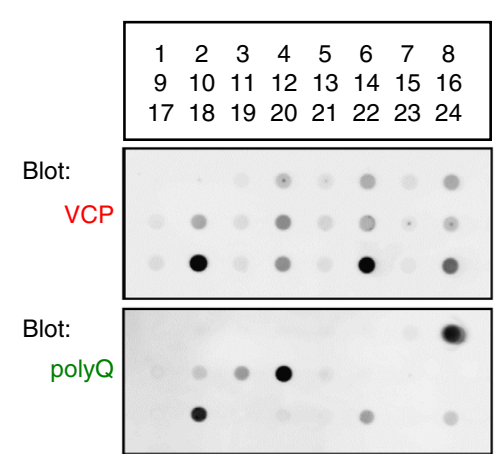

Figure 2 | Mutant polyQ proteins sequester TERA/VCP/p97 into the inclusion body. (a) HeLa cells co-transfected with Myc-polyQ and FLAG-VCP protein expression vectors are stained with anti-FLAG and anti-Myc antibodies. The top panels show normal cytoplasmic distribution of the FLAG-VCP protein. The ATXN1-86Q and Htt-103Q proteins sequester VCP to nuclear and cytoplasmic inclusion bodies, respectively. In contrast, the ATXN1-33Q and $\mathrm{Htt}-20 \mathrm{Q}$ proteins do not affect the cytoplasmic distribution of VCP regardless of their interaction. Normal and mutant ATXN7 expression did not affect intracellular localization of VCP. In the presence of testosterone, mutant but not normal AR sequesters VCP into the nucleus. Scale bar, $20 \mu \mathrm{m}$.

(b) Quantitative analysis of the change of intracellular distribution of VCP by polyQ proteins. The distribution patterns are described in right panels. Means and s.e.m. are indicated. Asterisks indicate statistical difference from the control $(P<0.01$, Student's $t$-test). (c) Filter-binding assay shows co-aggregation of polyQ disease proteins and VCP. 1, 2: buffer, 3 empty plasmid, 4: empty plasmid + VCP, 5: ATXN1-33Q, 6: ATXN1-33Q + VCP, 7: ATXN1-86Q, 8: ATXN186Q + VCP, 9: Htt-20Q, 10: Htt-20Q + VCP, 11: Htt-103Q, 12: Htt-103Q + VCP, 13: ATXN7-10Q, 14: ATXN7-10Q + VCP, 15: ATXN7-92Q, 16: ATXN7-

$92 \mathrm{Q}+\mathrm{VCP}, 17: \mathrm{AR}-24 \mathrm{Q}$ testosterone ( - ), 18: AR-24Q + VCP testosterone ( - ), 19: AR-24Q testosterone ( + ), 20: AR-24Q + VCP testosterone ( + ), 21: AR-64Q testosterone $(-), 22:$ AR-64Q + VCP testosterone $(-)$, 23: AR-64Q testosterone $(+), 24:$ AR-64Q + VCP testosterone $(+)$.

\section{Results}

Mutant and normal polyQ proteins interact with TERA/VCP/ p97. We performed an immunoprecipitation (IP) assay to test interaction between TERA/VCP/p97 and polyQ disease proteins. TERA/VCP/p97 was co-expressed in HeLa cells with full-length ataxin-1 (ATXN1), huntingtin exon 1 (Htt-exon1), full-length ataxin-7 (ATXN7) or androgen receptor (AR) by expression vectors. TERA/VCP/p97 was tagged with FLAG, while the polyQ proteins were tagged with Myc-peptide. Both anti-Myc and antiFLAG antibodies co-precipitated the counterpart (Fig. 1). The affinity was different for the various polyQ proteins; ATXN1 and Htt-exon1 were bound tightly to TERA/VCP/p97 (Fig. 1a,b), whereas interaction between ATXN7 and TERA/VCP/p97 seemed weak (Fig. 1a,b). However, beyond such differences, all polyQ proteins bound to TERA/VCP/p97. In the same IP condition, we could never found binding of c-Jun, c-Fos or RIP1 
a
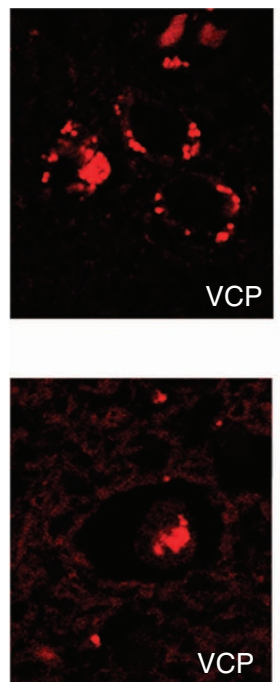

$\mathrm{CP}$
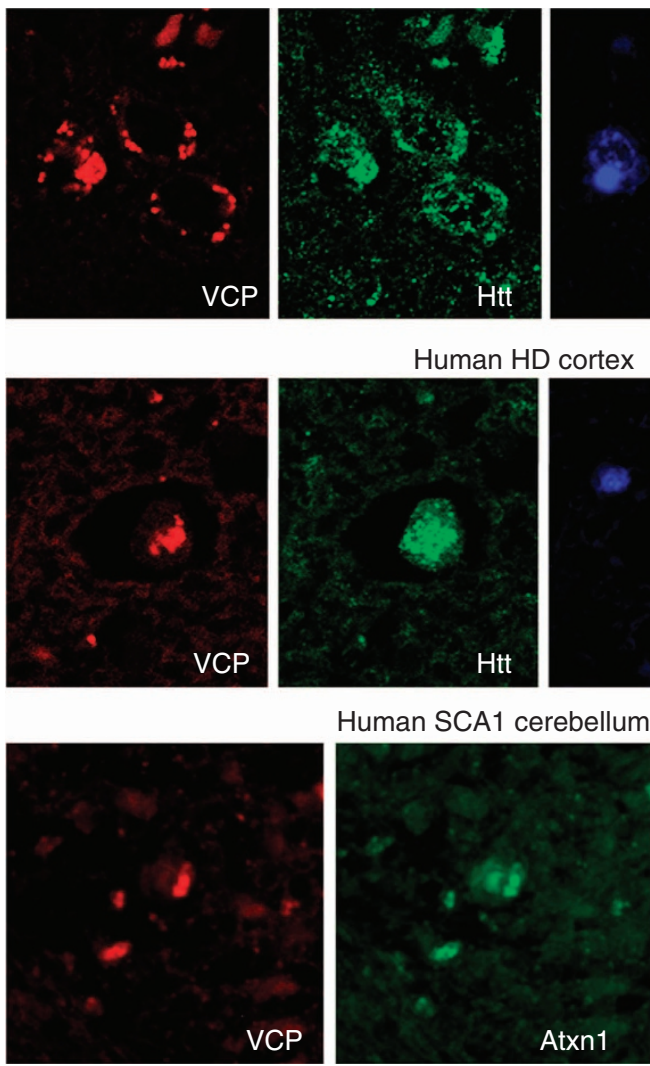

Human HD cortex
Human SCA1 cerebellum

Human HD caudate nucleus
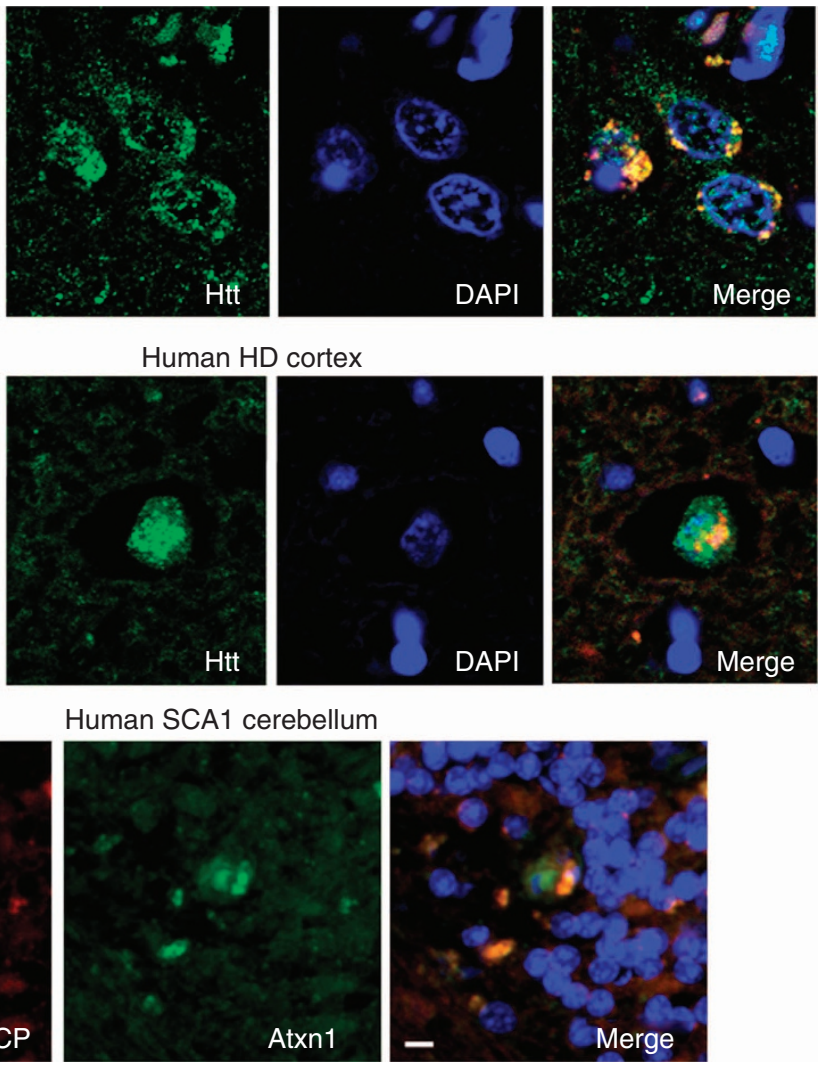

b

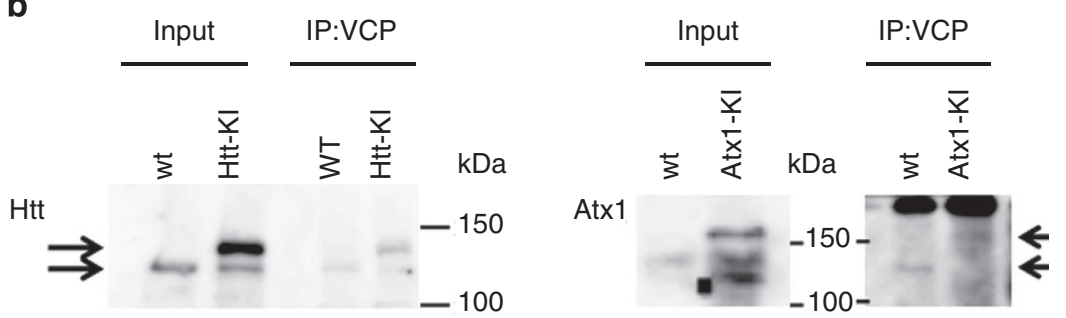

Figure 3 | Mutant polyQ proteins interact with TERA/VCP/p97 in the human brain. (a) Mutant polyQ proteins and VCP are co-localized in inclusion bodies of human patients with polyQ diseases. Subcellular distribution of VCP was remarkably changed in neurons possessing aggregates of polyQ disease proteins. Scale bar, $10 \mu \mathrm{m}$. In control human brains, subcellular accumulation or foci of TERA/VCP/p97 was not observed (Supplementary Fig. S6). (b) Coprecipitation of mutant polyQ and VCP proteins from mutant polyQ-KI mouse brain samples.

to polyQ proteins in multiple trials (Supplementary Fig. S1). Surprisingly, both normal as well as mutant polyQ proteins interacted with TERA/VCP/p97 in all instances, although the affinity of mutant polyQ protein was basically higher (Fig. 1a,b). The affinity to the soluble form of mutant polyQ protein was basically higher, except in the case of Htt-exon1-103Q protein where insoluble (bar in Fig. 1a) rather than soluble proteins (arrow in Fig. 1a) were sticky to TERA/VCP/p97. Multiple bands of polyQ proteins corresponded to different states of aggregation from monomer to polymer and/or different protein structures.

The interaction with $\mathrm{AR}$ was remarkably reduced in testosterone $(50 \mathrm{nM}, 24 \mathrm{~h})$-treated cells $(\mathrm{T}+)$, suggesting that nuclear translocation of ligand-bound AR decreases the chance of association with TERA/VCP/p97 localized to the cytoplasm in HeLa cells. Consistent with our previous observation ${ }^{3}$, deletion of the polyQ tract by using Htt, ATXN1, ATXN7 and AR remarkably decreased their affinities to TERA/VCP/p97 (Supplementary Fig. S2), indicating that interaction between polyQ and TERA/ $\mathrm{VCP} / \mathrm{p} 97$ is based on the polyQ tract sequence.
Mutant polyQ proteins mislocalize TERA/VCP/p97. We next addressed how interaction influences subcellular localization of TERA/VCP/p97. As neurons are not suitable for detailed analysis of cytoplasmic localization, we used HeLa cells with larger cytoplasm. Mutant ATXN1, AR(T + ) and Htt-exon1 proteins formed either nuclear or cytoplasmic inclusion bodies at high frequency (Fig. 2a). As expected from the biochemical interaction (Fig. 1), TERA/VCP/p97 was co-localized with most of the polyQ proteins (Fig. 2a). In such aggregate-positive cells, TERA/VCP/p97 was sequestered to the inclusion bodies (ATXN1-86Q, Htt-103Q and AR-64Q $\mathrm{T}+$ ) and its subcellular distribution was shifted (Fig. 2b). Filter-binding assay supported the sequestration of TERA/VCP/p97 by mutant polyQ proteins (Fig. 2c), and unbound TERA/VCP/p97 (= membrane filtered) was reduced by mutant polyQ proteins in a dose-dependent manner (Supplementary Fig. S3).

Distribution pattern of TERA/VCP/p97 was remarkably changed in cells co-expressing mutant ATXN1, AR or Htt-exon1 proteins (Fig. 2a,b), while mutant ATXN7 did not remarkably 
a

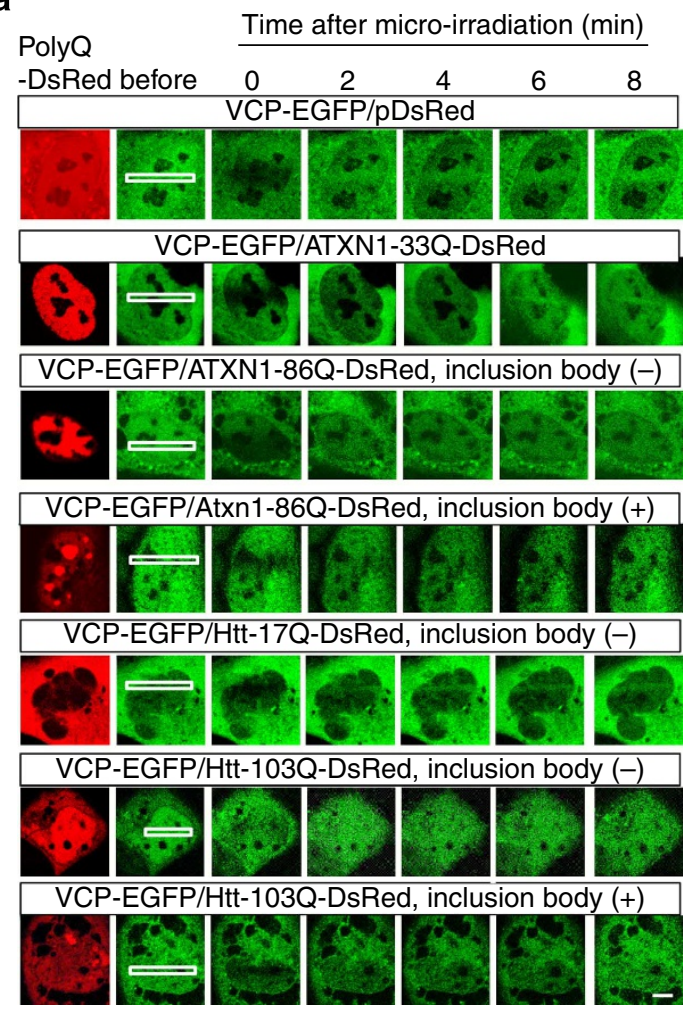

b
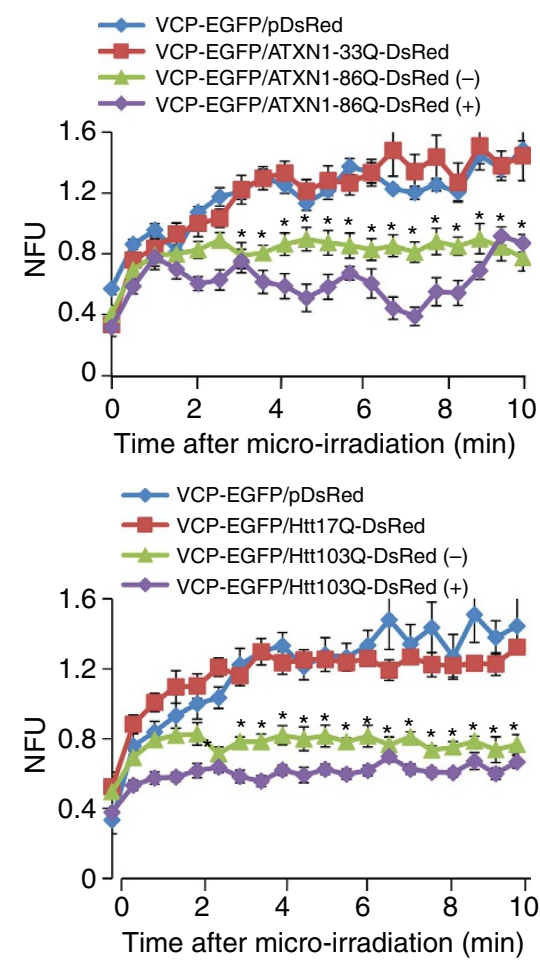

C

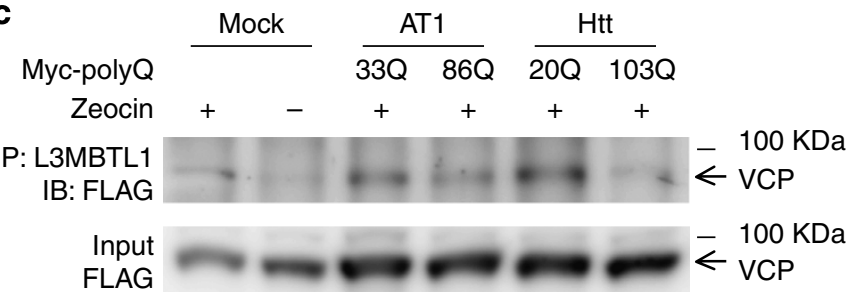

Figure 4 | Mutant polyQ proteins delay accumulation of TERA/VCP/p97 to DSB foci. (a) Sequential images showing accumulation of VCP-EGFP to the linear DSB. In cells expressing mutant polyQ proteins (Atxn1-86Q-DsRed and Htt-103Q-DsRed), the accumulation of VCP-EGFP was delayed in comparison with non-transfected or normal polyQ protein (Atxn1-33Q-DsRed and Htt-17Q-DsRed) expressing cells. Among the cells expressing mutant polyQ proteins, the delay in the accumulation of VCP-EGFP was more remarkable in inclusion body-positive cells than in inclusion body-negative cells. Scale bar, $5 \mu \mathrm{m}$. (b) Quantitative analysis of the recovery of VCP-EGFP signals at the linear photobleach areas that correspond to accumulation of VCP-EGFP to the DSB lesion $(N=6)$. The signals were obtained from more than four sites on the linear damage area, and the mean value was used for the quantitative analyses at each time point. Means and s.e.m. are indicated. Asterisks indicate statistical difference from the value of normal polyQ protein-expressing cells at the same time point $(P<0.01$, Student's $t$-test). H2Ax staining confirmed that DSB was actually induced in this condition (Supplementary Fig. 8). NFU: normalized fluorescence unit. (c) Mutant polyQ proteins inhibit interaction between TERA/VCP/p97 and L3MBTL1. Zeocin $\left(400 \mu \mathrm{g} \mathrm{ml}^{-1}\right.$ ) was added to the culture medium of HeLa cells to induce DSBs where TERA/VCP/p97 interacts with L3MBTL1. Mutant Htt and ATXN1 both decrease the interaction between TERA/VCP/p97 and L3MBTL1.

shift TERA/VCP/p97 to the nucleus (Fig. 2a,b) agreeing with the weak binding between ATXN7 and TERA/VCP/p97 (Fig. 1a,b). It is of note that TERA/VCP/p97 in the nucleoplasm was substantially reduced in mutant ATXN1- or mutant Htt-expressing cells (Fig. 2a). In the case of mutant ATXN1-expressing cells, TERA/VCP/p97 was sequestered to nuclear inclusions and deprived from the nucleoplasm (Fig. 2a), while the deprivation was unclear in mutant $\mathrm{AR}(\mathrm{T}+)$ cells with homogeneous nuclear aggregates (Fig. 2a).

Meanwhile, intracellular distribution of TERA/VCP/p97 was not changed by normal polyQ proteins (Fig. 2a,b); instead, it was consistent with a low affinity to TERA/VCP/p97 in the case of ATXN7 or with the cytoplasmic non-aggregating localization of the normal $\mathrm{AR}(\mathrm{T}-)$ and Htt-exon1. However, in the case of normal ATXN1 and $\operatorname{AR}(\mathrm{T}+)$ located in the nucleus, sequestration of TERA/VCP/p97 was not observed, despite the biochemical interaction. This might be due to a difference in biochemical composition between nuclear foci and nuclear inclusion bodies. Even in the case of nuclear inclusion bodies, reports have shown that polyQ proteins dynamically shuttle in and out of the inclusion bodies and that the shuttling speed is dependent on polyQ length ${ }^{25-27}$. Presumably, sequestration can occur only when TERA/VCP/p97 is bound tightly to the inside structure of such aggregates.

Mutant polyQs impair DSB repair function of TERA/VCP/ p97. TERA/VCP/p97 have multiple functions like protein transport from ER to the plasma membrane, ERAD and recently identified DSB repair ${ }^{13,14}$. Surprisingly, immunohistochemistry 
a

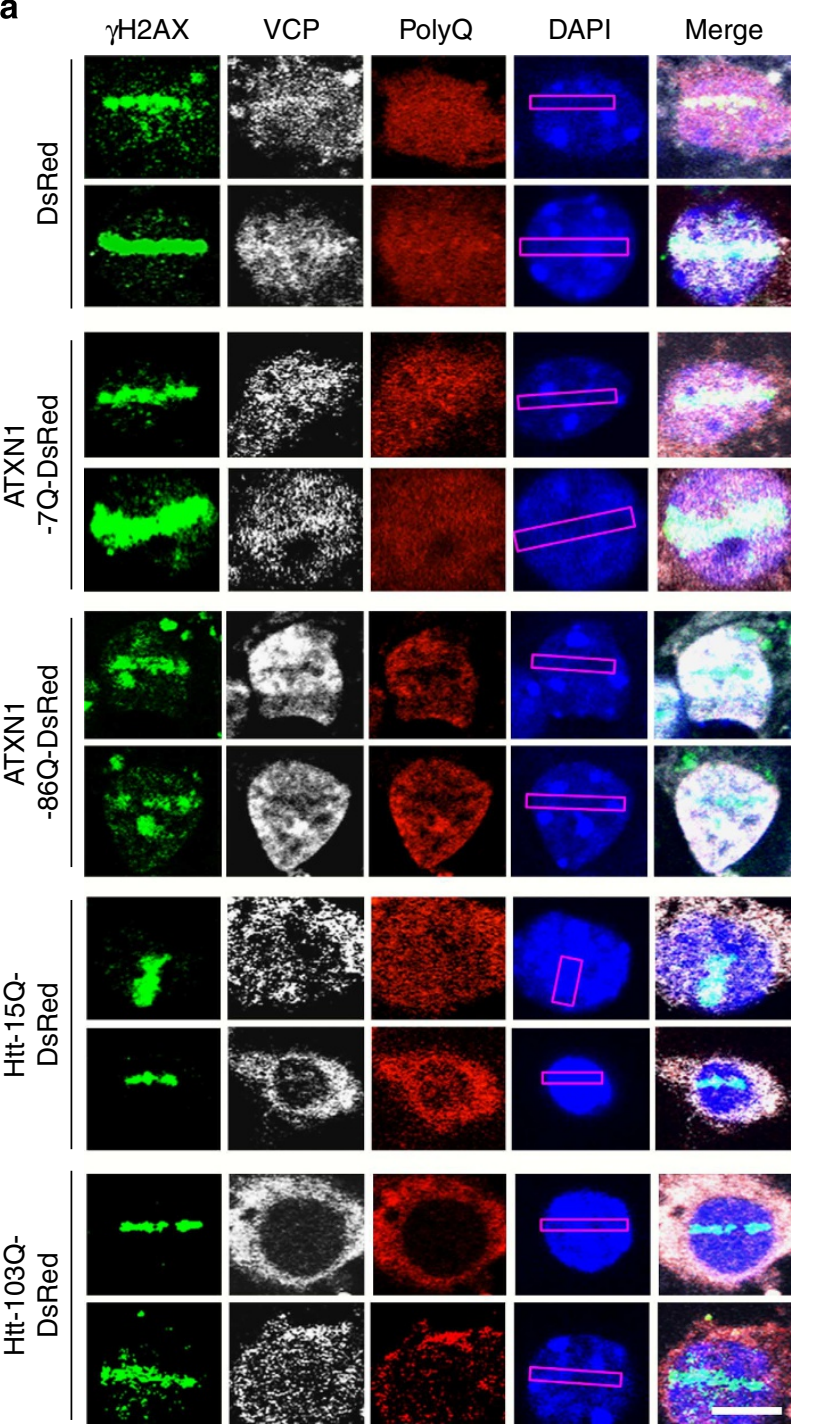

b

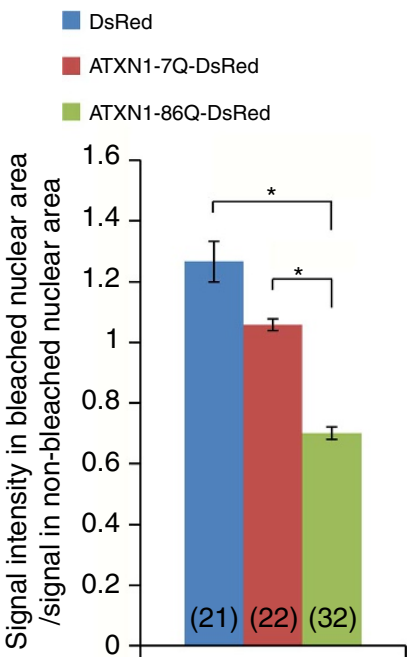

DsRed

Htt-15Q-DsRed

Htt-103Q-DsRed

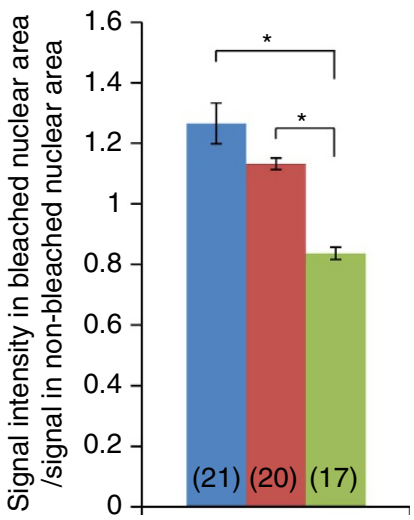

Figure 5 | Delayed dynamics of TERA/VCP/p97 recruitment to DSBs by mutant polyQ proteins in primary cortical neurons. (a) The fluorescent signal of TERA/VCP/p97 at DSBs was decreased in Atxn1-86Q-DsRed or Htt-103Q-DsRed expressing mouse primary cortical neurons more than the control neurons expressing DsRed, Atxn1-7Q-DsRed or Htt-15Q-DsRed at $1 \mathrm{~h}$ after laser microirradiation. Signals of TERA/VCP/p97 were obtained from $\gamma \mathrm{H} 2 \mathrm{AX}$ positive bleached area. Scale bar, $5 \mu \mathrm{m}$. (b) The signal intensity in bleached nuclear area/signal in non-bleached nuclear area was decreased in mutant polyQ expressing neurons more than the control neurons. Means and s.e.m. are indicated. Asterisks indicate statistical difference of the value between control neurons and polyQ protein-expressing neurons $(P<0.01$, Student's $t$-test).

with multiple anti-VCP antibodies revealed that TERA/VCP/p97 is localized dominantly in the nucleus of adult neurons (Supplementary Fig. S4) contrastively to the cytoplasmic localization in hepatocytes or renal tubules cells (Supplementary Fig. S5). Furthermore, the nuclear dominant distribution of TERA/VCP/p97 was confirmed in normal human neurons including vulnerable subtypes in polyQ diseases (Supplementary Fig. S6). The result clearly indicated that nuclear function of TERA/VCP/p97 is more important than the other cytoplasmic functions, in human and mouse adult neurons.

Therefore, we firstly tested whether sequestration of TERA/ VCP/p97 impairs DSB repair. Immunohistochemistry with human patient brains of $\mathrm{HD}$ and SCA1 revealed that TERA/ $\mathrm{VCP} / \mathrm{p} 97$ was depleted from the nucleoplasm in affected neurons by sequestration to cytoplasmic (perinuclear) or nuclear inclusion bodies (Fig. 3a). In human HD brains, TERA/VCP/p97 was colocalized with mutant $\mathrm{Htt}$ in the marginal zone of the nucleus or in the perinuclear cytoplasmic region of striatal neurons (Fig. 3a, upper panels); however, TERA/VCP/p97 was sometimes concentrated in the cleft, where the cytoplasm is inserted into the nucleus in cortical neurons (Fig. 3a, middle panels). In human SCA1 brains, TERA/VCP/p97 was co-localized in nuclear inclusions of Purkinje cells or occasionally in shrunken nuclei of cerebellar granule cells (Fig. 3a, lower panels). In all cases, signals of TERA/VCP/p97 were very low in the nucleoplasm.

Interaction between polyQ proteins and TERA/VCP/p97 in vivo and sequestration of TERA/VCP/p97 into aggregates were also confirmed by IP (Fig. 3b) and immunohistchemsitry (Supplementary Fig. S7) with brains from model mice. We used KI mouse brain tissues from full-length mutant $\mathrm{Htt}-111 \mathrm{Q}^{28}$ or mutant ATXN1-154Q ${ }^{29}$, together with mutant Htt- ${ }^{30}$, ATXN7-31 or AR-transgenic (Tg) mice ${ }^{32}$. Again we confirmed the co-localization of mutant polyQ proteins with TERA/VCP/p97 in Htt-Tg (R6/2), Htt-KI, ATXN1-KI and AR-Tg mice (Supplementary Figs S7a-c, S8). In ATXN7-Tg mice in which ATXN7 is expressed just above the endogenous level ${ }^{31}$, sequestration of VCP 


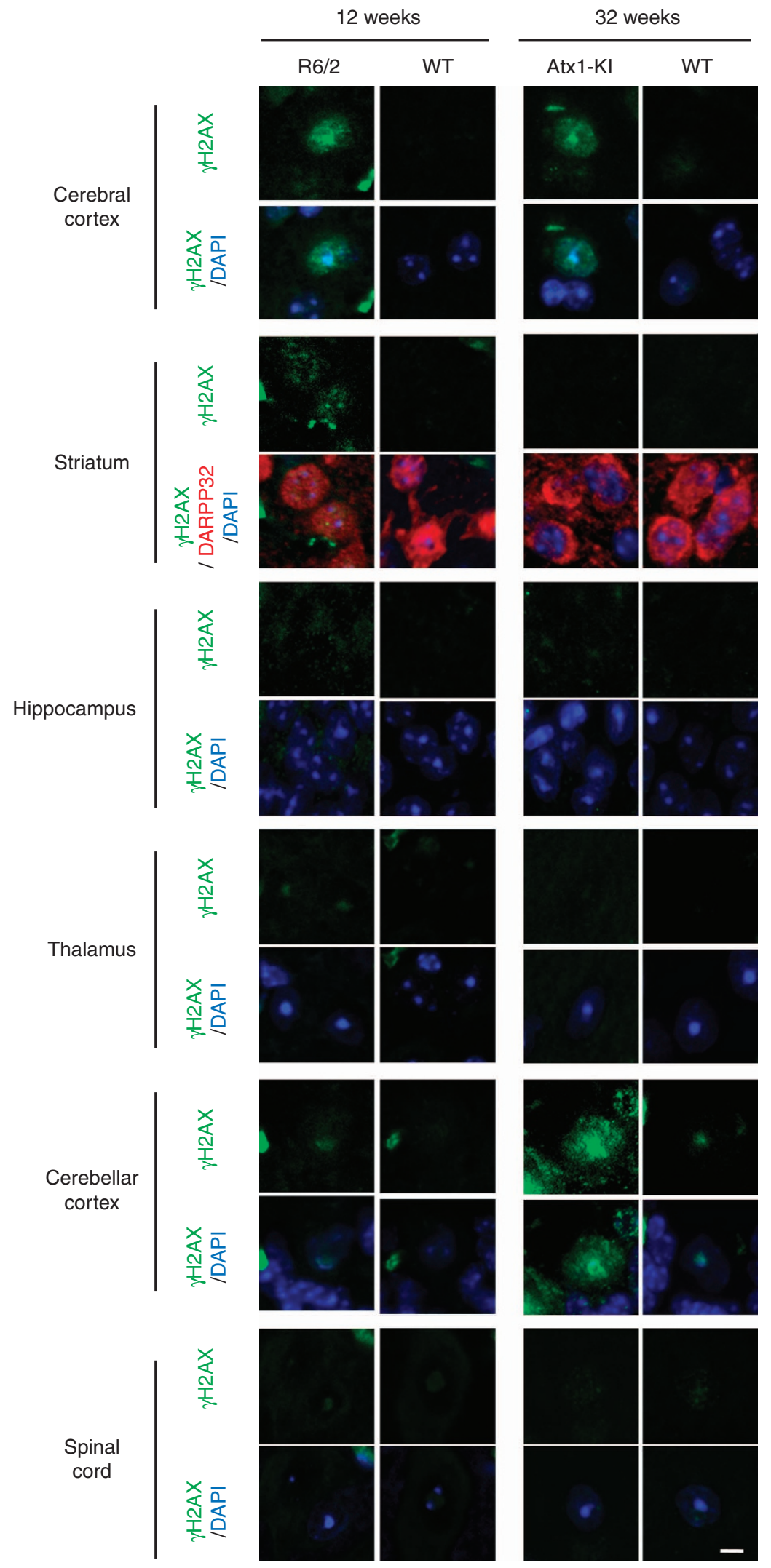

Figure 6 | DSBs are prominent in vulnerable types of neurons. Various brain regions of R6/2 (12 weeks) and Atxn1-KI mice (32 weeks) were stained with $\gamma \mathrm{H} 2 \mathrm{AX}$ antibody. The controls (WT) were age-matched littermates. DARPP32 is a marker of medium spiny neurons. Scale bar, $5 \mu \mathrm{m}$.

to the inclusion body was not obvious (Supplementary Fig. S7d). In Htt-Tg (R6/2), Htt-KI, ATXN1-KI and AR-Tg mice, TERA/VCP/ p97 was decreased in the nucleoplasm when compared with their controls (Supplementary Fig. S8). An IP assay also confirmed endogenous interaction between mutant $\mathrm{Htt}$ and TERA/VCP/p97 in HD model mice (Fig. 3b). 
Moreover, we tested whether mutant polyQ proteins impair the dynamics of TERA/VCP/p97 to DSB lesions induced by highenergy UVA (Supplementary Fig. S9). In U2OS cells expressing Atxn1-86Q-DsRed, accumulation of VCP-EGFP to the DSB lesion was slowed down in comparison with Atxn1-33Q-DsRedexpressing cells or mock-treated cells (Fig. 4a,b). In the case of mutant Htt expression, the delay was also remarkable in the cells possessing DsRed signals of $\mathrm{Htt}-103 \mathrm{Q}$ in the nucleus even without nuclear inclusion body (Fig. 4a,b), suggesting that pre-aggregates of mutant $\mathrm{Htt}$ impaired dynamics of TERA/VCP/p97 to nuclear DSB lesions. However, the delay of VCP-EGFP accumulation became more profound when inclusion bodies were formed in Atxn1-86QDsRed or Htt-103Q-DsRed expressing cells (Fig. 4a,b), indicating that aggregation also contributes to the delay.

We also tested the TERA/VCP/p97 dynamics after microirradiation in primary mouse cortical neurons. As primary neurons have too small nuclei for high-energy damage and they were highly vulnerable to suffer some levels of DNA damages during primary culture, we used lentiviral vectors for expression of polyQ proteins, reduced the energy of microirradiation and performed signal acquisition by immunocytochemistry at $1 \mathrm{~h}$ after microirradiation. Accumulation of TERA/VCP/p97 to damaged area was also delayed in primary neurons expressing Atxn1-86Q-DsRed or Htt-103Q-DsRed in comparison with primary neurons expressing DsRed or normal polyQ proteins (Fig. 5a,b).

TERA/VCP/p97 interacts with L3MBTL1, facilitates replacement of L3MBTL1 with 53BP1, changes the chromatin for DSB repair ${ }^{13}$. Therefore, we tested whether mutant polyQ proteins disturbed this process. Consistently with the impaired dynamics of TERA/VCP/ p97 in the nucleus, both ATXN1-86Q and Htt-103Q proteins inhibited interaction between transfected VCP-FLAG and endogenous L3MBTL1 (Fig. 4c). Moreover, extraction of L3MBTL1 from the linear DSB lesion and replacement with 53BP1 were actually delayed at an early time point after microirradiation (Supplementary Fig. S10a,b). Consequently, the signal intensities of $\gamma \mathrm{H} 2 \mathrm{Ax}$ remained at higher levels in U2OS cells expressing Atxn1-86Q-DsRed or Htt-103Q-DsRed (Supplementary Fig. S10c).

The nuclear situation in U2OS cells could be analogous to polyQ disease mouse models in which TERA/VCP/p97 was deprived from nucleoplasm to inclusion bodies (Supplementary Figs 7,8). It could explain the remarkable increase of DSB in polyQ disease mouse models (Supplementary Fig. S11). High $\gamma \mathrm{H} 2 \mathrm{Ax}$ signals were observed predominantly in vulnerable neurons of each mouse model (Fig. 6) and correlated with sequestration or disappearance of TERA/VCP/p97 (Supplementary Fig. S12).

Mutant polyQs affect ER-related functions of TERA/VCP/p97. Although the nuclear function of TERA/VCP/p97 was implicated in the polyQ disease pathology, involvement of the other functions of TERA/VCP/p97 cannot be excluded. Therefore, we tested whether mislocalization or sequestration influenced the other cytoplasmic functions of TERA/VCP/p97 like protein transport and ERAD. In the first case, dominant-negative TERA/VCP/p97 (VCP-DN) was known to impair transport of ErbB2 protein from ER to the plasma membrane ${ }^{33}$. They reported that ErbB2 could not pass the quality control system and accumulated around the nucleus $^{33}$. As expected, when mutant polyQ proteins were expressed in HeLa cells, EGFP-ErbB2 was stacked in the perinuclear region (Supplementary Fig. S13a). AR-64Q induced similar accumulation irrespective of testosterone (Supplementary Fig. S13a). TERA/VCP/p97 co-expression recovered the ErbB2 perinuclear accumulation (Supplementary Fig. S13b,c). Mutant polyQ proteins also increased cytoplasmic vacuoles co-stained with an ER marker but distinct from autophagosomes (Supplementary Fig. S14a,b).

Moreover, we analysed primary mouse cortical neurons transfected by polyQ-DsRed and VCP-EGFP expression vectors. Co-localization of mutant polyQ and TERA/VCP/p97 proteins was confirmed (Supplementary Fig. S15) like human or mouse disease brains (Supplementary Figs S6, 7). In DsRed expressing neurons, VCP-EGFP was homogenously localized in the nucleus, while expression of Atxn1-86Q-DsRed changed the distribution heterogeneous in the nucleus and partially shifted to the cytoplasm (Supplementary Fig. S15). Htt-103Q-DsRed clearly sequestered VCP-EGFP to inclusion bodies (Supplementary Fig. S15). We also found that EGFP-ErbB2 was stacked around the nucleus in a part of primary neurons expressing mutant polyQ proteins (Supplementary Fig. S16).

Next, we investigated the effect of polyQ proteins on ERADan important function of TERA/VCP/p97-by using HEK293 cells and an ERAD substrate, CD3 $\Delta$. Expression vectors for $\mathrm{Htt}$ and ATXN1 proteins were co-transfected. When protein synthesis was blocked with cycloheximide (CHX), the actual amount of CD3 $\Delta$ degradation by ERAD could be visualized (Supplementary Fig. S17). Mutant but not normal Htt protein inhibited CD3 $\Delta$ degradation significantly, whereas co-expression of TERA/VCP/p97 reverted CD3 $\Delta$ accumulation by mutant $\mathrm{Htt}$ (Supplementary Fig. S17a-c, left panels). Similar findings were observed in HeLa cells expressing the mutant ATXN1 protein (Supplementary Fig. S17a-c, right panels).

Further, to test the effect of mutant polyQ proteins on ERAD, we used primary cortical neurons prepared from Htt-111Q or ATXN1-154Q knock-in (KI) mice and tested CD3 $\Delta$ degradation at 100 or $300 \mu \mathrm{g} \mathrm{ml}^{-1} \mathrm{CHX}$ (Supplementary Fig. S18). We confirmed that mutant $\mathrm{Htt}$ or ATXN1 expression disturbed ERAD at the endogenous expression level (Supplementary Fig. S18a-c); however, the effect was smaller than that observed in HeLa cells.

Mutant polyQs weakly inhibit TERA/VCP/p97 ATPase activity. We next determined whether mutant polyQ proteins affect the ATPase activity of TERA/VCP/p97. To do this, we used KI mice with the full-length human mutant Htt-111Q gene. We prepared from brain samples high molecular weight fractions corresponding to TERA/VCP/p97 immunoreactivity (Supplementary Fig. S18d). The fractions preserve the native hexamer of TERA/VCP/p97, which differs from the reconstructed TERA/VCP/p97 fusion protein produced in Escherichia coli cells, and we used it for an in vitro ATPase activity assay ${ }^{22,34}$. In this direct assay, ATPase activity of TERA/VCP/p97 per brain tissue weight was decreased in mutant Htt-KI mice (Supplementary Fig. S18d).

We then examined the complex formation of TERA/VCP/p97 and Derlin-1 for protein quality control. According to the method described previously ${ }^{9}$, we tested the complex formation with brain samples from mutant Htt-KI mice. As expected, Derlin-1 co-precipitation with TERA/VCP/p97 in cortex was reduced (Supplementary Fig. S18e) indicating that interaction of TERA/ $\mathrm{VCP} / \mathrm{p} 97$ with mutant Htt inhibits the complex formation with Derlin-1.

Taken together, the scheme obtained here was novel, and distinct from the previous one. This study revealed that mutant polyQ proteins suppressed ATPase activity of TERA/VCP/p97 in the brain tissue in vivo in contrast to the previous concept. Our results also suggested that non-cytoplasmic function of TERA/ $\mathrm{VCP} / \mathrm{p} 97$, that is, nuclear function of TERA/VCP/p97 is more critical for neurodegeneration. Mutant polyQ proteins suppressed dynamics of the nuclear TERA/VCP/p97 essential for DNA repair 
a

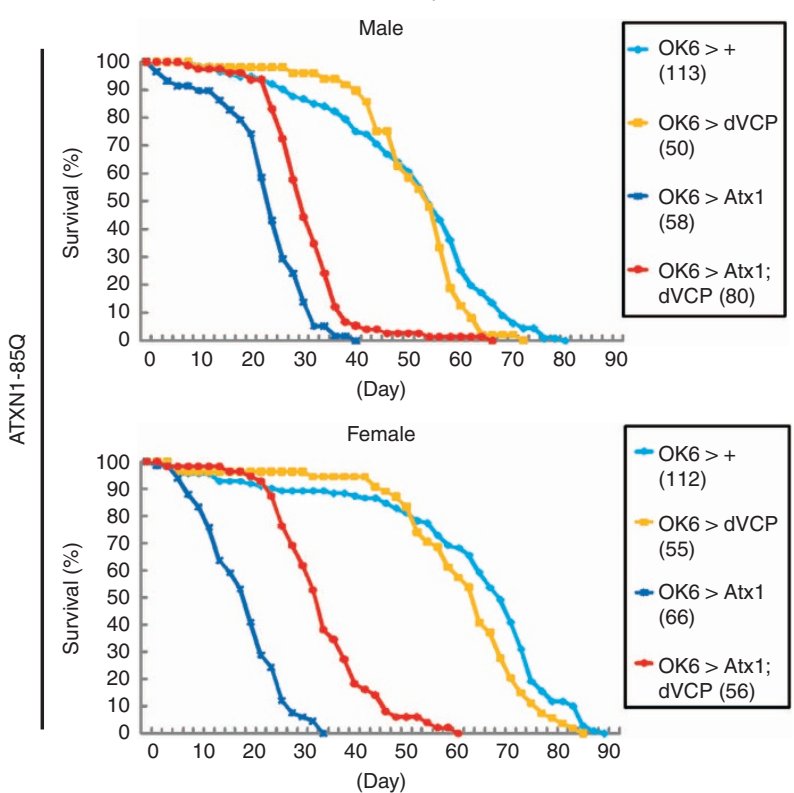

b

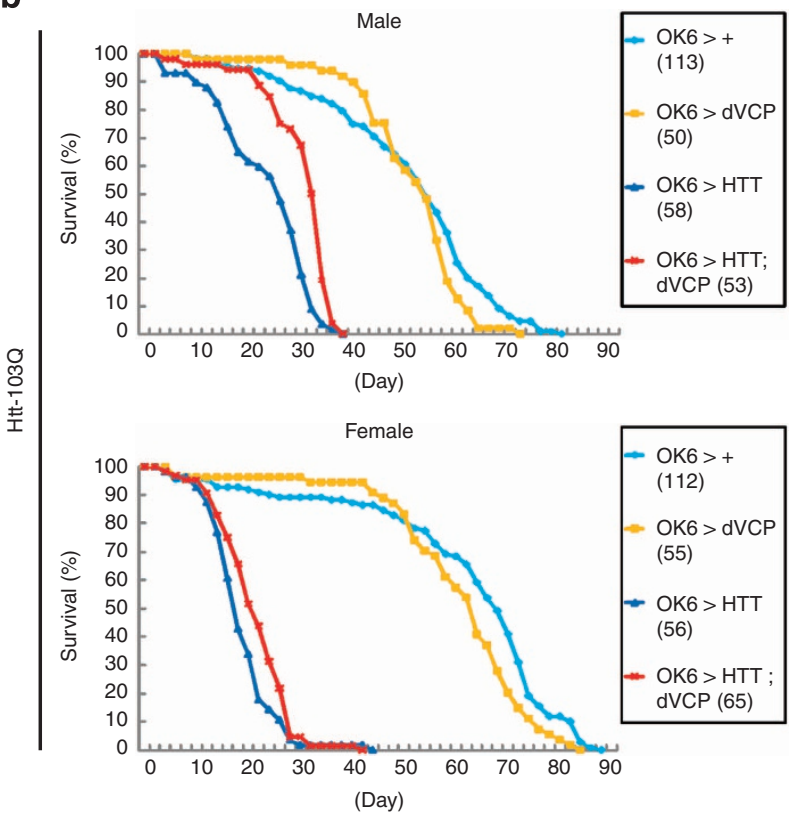

Rescue of DSB
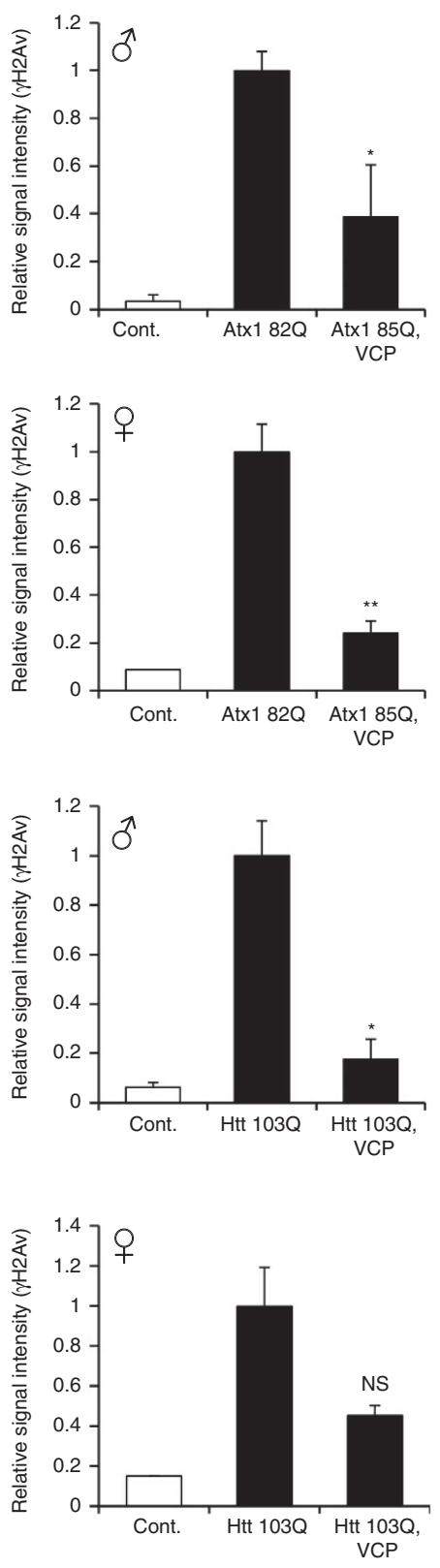

Figure 7 | TERA/VCP/p97 rescues lifespan shortening in mutant Htt and ATXN1 transgenic flies. Human Atxn1-85Q full-length protein (a) or Human $\mathrm{Htt}-103 \mathrm{Q}$-exon1 peptide (b) expression in motor neurons by OK6-driver shortens the lifespan. Co-expression of VCP elongates the lifespan of both Atxn1 and $\mathrm{Htt}$ transgenic flies (log-rank test: $P<0.01$ in Atxn1 males and females and $\mathrm{Htt}$ males). The numbers of flies are shown after each fly genotype. Right graphs show the signals of $\gamma \mathrm{H} 2 \mathrm{Av}$ indicating double strand break (DSB) DNA damages. The signals were obtained from thoracic motor neurons ( $n=10-$ 30/slide, 1 slide/fly, 5 flies/genotype), subtracted with background signals, and corrected by the number of neurons. The relative values were calculated as the ratio to the signals of mutant Atxn1 or Htt transgenic flies. Means and s.e.m. are indicated. ${ }^{\star} P<0.05$, Student's $t$-test, ${ }^{\star \star} P<0.01$, Student's $t$-test.

(Fig. 4, Supplementary Fig. S10) and increased DNA damage (Fig. 6, Supplementary Fig. S11).

TERA/VCP/p97 rescues polyQ phenotypes in Drosophila. Finally, we analysed whether TERA/VCP/p97 can rescue neurodegeneration phenotypes in vivo. For this analysis, we employed a new Drosophila model in which polyQ proteins are expressed specifically in motor neurons by the OK6 driver ${ }^{35}$. We used the GAL4-UAS system in which a transposon-containing upstream activation sequence (UAS) cis-element linked to a human polyQ gene is randomly integrated into the genome. We created UASHtt-103Q and UAS-ATXN1-85Q transgenic flies possessing a transgene integrated into chromosome 2. In the fly models, lifespan was shortened remarkably (Fig. 7). We then mated the flies with TERA/VCP/p97 transgenic flies and observed the effect on the lifespan shortening. Consequently, TERA/VCP/p97 expression recovered the lifespan shortening of ATXN1-85Q and Htt-103Q transgenic flies (Fig. 7). We confirmed equivalent expression levels of polyQ genes in the disease model and rescue flies by quantitative PCR (Supplementary Fig. S19a). Moreover, TERA/VCP/p97 restored the reduced thickness of the complex eye of the GMRGal4/UAS-ATXN1 fly (Supplementary Fig. S19b,c).

Immunohistochemstry of motor neurons revealed that the extent of phenotype recovery was correlated well with the severity 

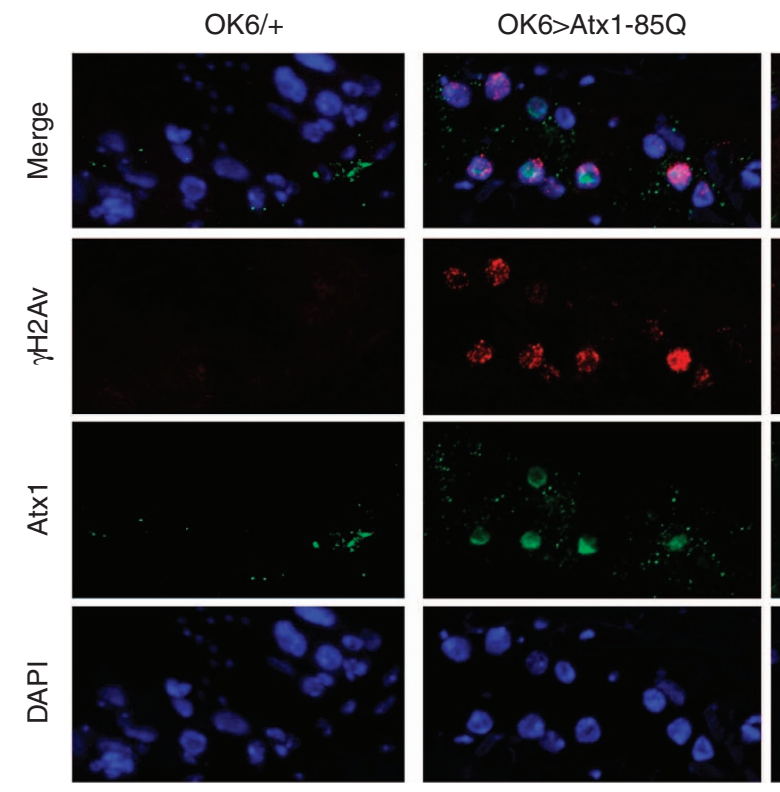

OK6>Atx1-85Q, VCP
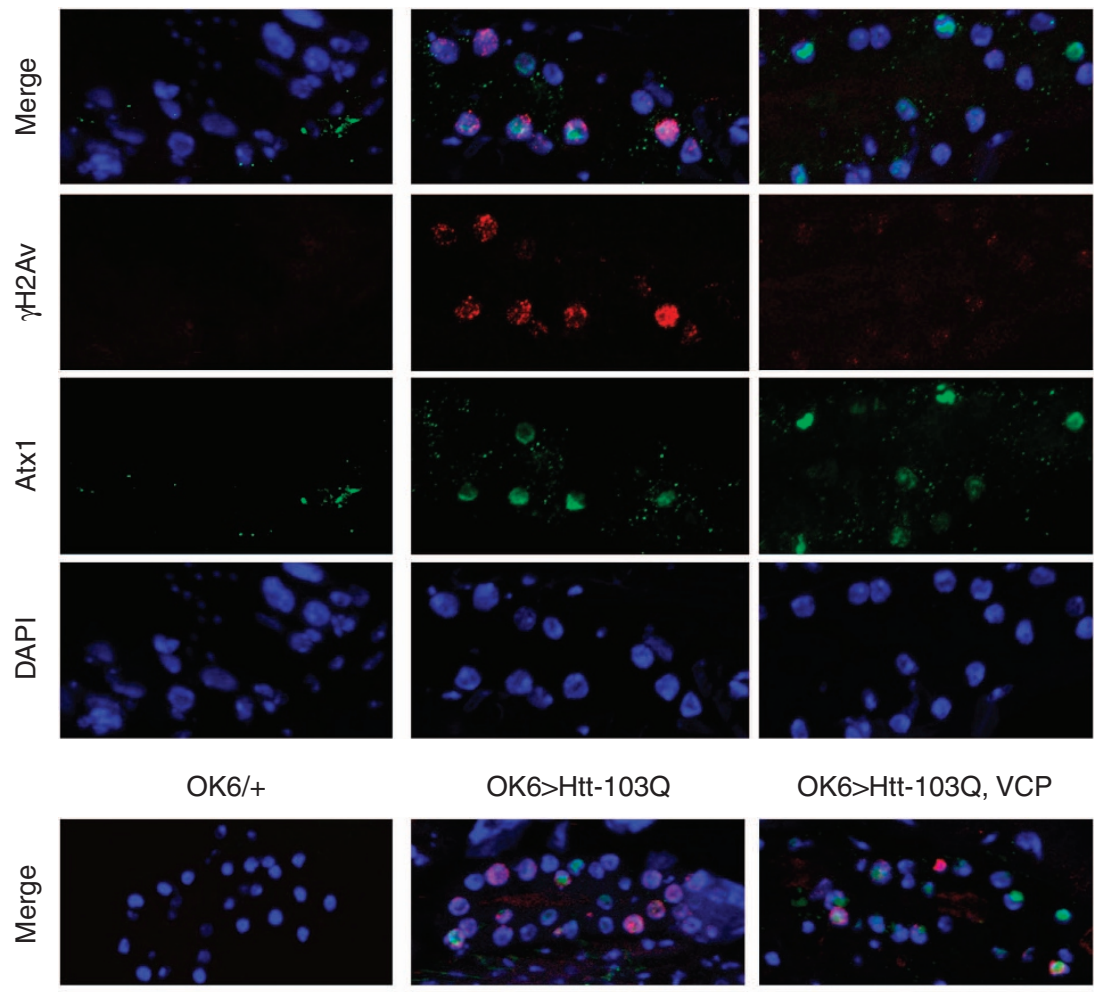

OK6>Htt-103Q, VCP
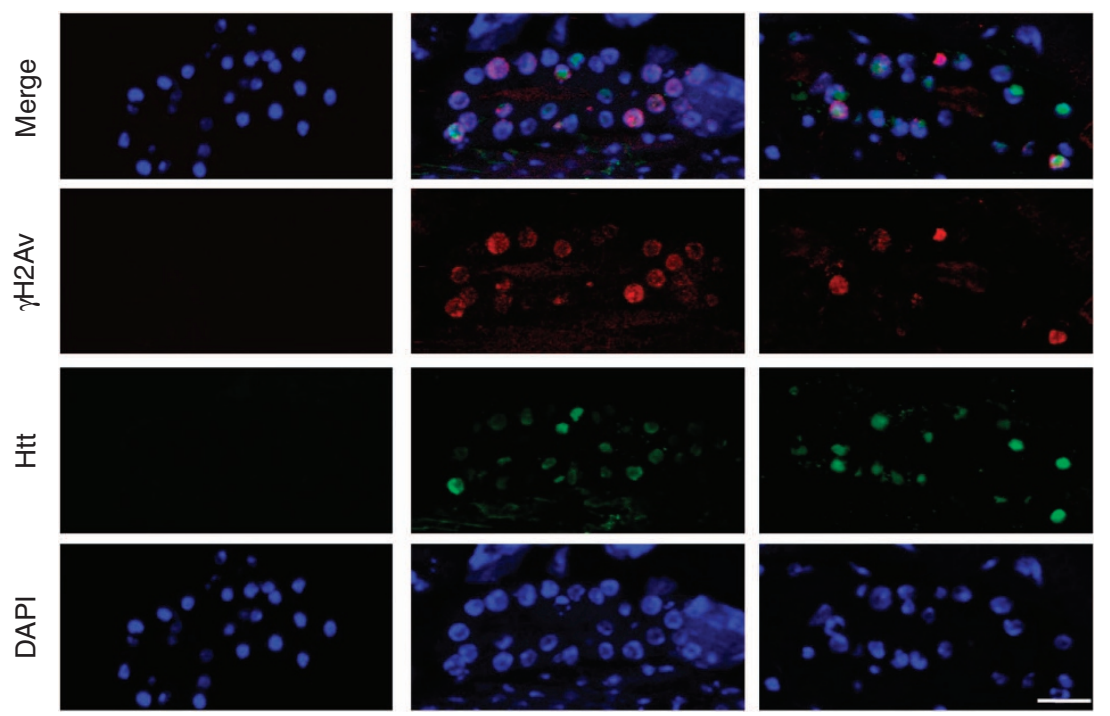

Figure 8 | TERA/VCP/p97 recovers DSBs in Drosophila motor neurons without affecting inclusion body formation. Drosophila thoracic motor neurons were co-stained with anti- $\gamma \mathrm{H} 2 \mathrm{Av}$ and anti-Atxn1 $(\mathrm{H} 21)$ or anti- $\mathrm{Htt}(\mathrm{N}-18)$ antibodies. DSB detected by anti- $\gamma \mathrm{H} 2 \mathrm{Av}$ antibody was recovered by co-expression of VCP in SCA1 or Htt fly models, while inclusion body formation of mutant Htt or diffuse nuclear accumulation of mutant ATXN1 was not remarkably changed. Scale bar, $10 \mu \mathrm{m}$.

of DSB evaluated by staining with anti- $\gamma \mathrm{H} 2 \mathrm{Av}$ antibody in SCA1 fly models (Fig. 7 right graph, Fig. 8). On the other hand, inclusion body formation of mutant $\mathrm{Htt}$ or diffuse nuclear accumulation of mutant ATXN1 was not largely affected by TERA/VCP/p97 (Fig. 8).

Collectively, our results support that functional defect of TERA/ $\mathrm{VCP} / \mathrm{p} 97$ in DSB repair is a major factor contributing to neurodegeneration induced by mutant polyQ proteins in vivo.

\section{Discussion}

TERA/VCP/p97 is a multi-functional protein implicated in membrane traffic, cell cycle regulation, nuclear envelope reconstruction, ERAD, DNA damage repair and and autophagy ${ }^{5-14,18}$. Among such multiple functions, results in this study strongly suggest that functional defect of TERA/VCP/p97 in DSB repair underlies multiple polyQ diseases (Supplementary Fig. S20). Mutant polyQ proteins impaired DSB repair by disturbing dynamics of TERA/VCP/p97 to DSB foci (Fig. 4). Moreover, nuclear distribution of TERA/VCP/p97 in human adult neurons (Supplementary Fig. S6), its sequestration into aggregates in human polyQ disease brains (Fig. 3), and the tight link between in vivo phenotypes and DSBs (Fig. 6) strongly supported that DSB repair mainly contributes to TERA/VCP/p97-mediated pathology in adult neurons.

We reported previously that DNA repair molecules, HMGB1/2 and $\mathrm{Ku} 70$, were involved in $\mathrm{HD}$ and $\mathrm{SCA1} 1^{36,37}$, which has recently been supported by the discovery of macroH $2 \mathrm{~A}-\mathrm{a}$ human biomarker of $\mathrm{HD}^{38,39}$. This study added another DNA repair molecule, TERA/VCP/p97 to the pathology and further strengthened the DNA damage repair hypothesis of $\mathrm{HD}$.

In addition to TERA/VCP/p97's function in DSB repair, some nuclear functions of TERA/VCP/p97 have been suspected. TERA/ $\mathrm{VCP} / \mathrm{p} 97$ interacts with the Aurora B kinase and regulates the reformation of the nucleus during the cell cycle ${ }^{40}$, and it might 
also act as a protein aggregation sensor that moves to the nucleus in response to protein aggregation ${ }^{24}$. As activation of cell cycle in neurodegeneration has been suggested by a number of results in non-dividing neurons ${ }^{41-43}$, our results do not exclude contribution of such nuclear functions of TERA/VCP/ p97 to the polyQ disease common pathology. Presumably, such nuclear functions of TERA/VCP/p97 could be involved in the polyQ disease pathology, and further studies are necessary in the future.

An increasing number of reports suggest that multiple polyQ diseases are partially based on common pathologies but also dependent on unique pathologies specific to each polyQ disease. Intriguingly, recent progress in interactome analysis and Drosophila genetics has contributed to this concept of common and unique pathologies ${ }^{37,44-46}$. Interactome analyses suggested that causative genes for polyQ diseases have mutually close relationships (http://string-db.org/) - a finding that was confirmed by genetic interaction in lower animal models ${ }^{44}$. Furthermore, comparison of transcriptome analysis data of multiple SCAs showed common signalling pathways ${ }^{47}$. This study supported the hypothesis through adding a new key player to the defect of DNA damage repair that has been suggested as a common pathology of polyQ diseases.

Beyond clarification of a new foci of common polyQ pathologies by TERA/VCP/p97, this study even suggest a novel common pathology that could be shared by a wider range of neurodegenerative disorders, including polyQ diseases and TERA/VCP/p97-linked FTLD. Ubiquitin and TDP43-positive inclusion bodies are observed in the nuclei of cortical neurons in FTLD with TERA/VCP/p97 mutations ${ }^{48}$. The nuclear or cytoplasmic aggregates might disturb dynamics of TERA/VCP/ p97 in the nuclei of neurons necessary for DSB repair, although the hypothesis should be further tested in the future.

Again our results cast a light on the role of inclusion body because TERA/VCP/p97 was deprived by aggregation. The pathological significance of inclusion bodies or aggregation has been a focus of debate for over a decade. The previous concept that the inclusion body by itself possesses toxicity has been challenged after the observation that inclusion body-positive neurons are more resistant to mutant $\mathrm{Htt}^{49}$. We also observed that cell death is inversely related to inclusion bodies in primary neurons expressing mutant $\mathrm{Htt}^{50}$. However, the pathological role of the inclusion body remains ambiguous, because misfolded protein toxicity stemming from abnormal physiological protein interactions are inseparable from the aggregating structures of a disease protein.

In our study, mutant polyQ proteins generally reduce the amount of TERA/VCP/p97 in the functional domains in cells, polyQ protein-mediated sequestration may also contributes to VCP pathology, at least as part of the disease process. These data seemed to support the later view. This idea was supported by the observation that the TERA/VCP/p97 dynamics in the nucleus was profoundly disturbed in inclusion body-positive cells (Fig. 4). However, soluble protein or pre-aggregates of mutant polyQ proteins could also be toxic to the functions of TERA/VCP/p97. Actually, our live imaging showed a delay of TERA/VCP/p97 accumulation to DSB lesions even in cells without inclusion body (Fig. 4). Moreover, the phenotype recovery of SCA1 and HD model flies was independent of aggregation (Fig. 8). The discussion has not reach to a definite conclusion in this study as in many other cases of polyQ disease models, and probably both mechanisms will contribute to the pathology.

Finally, although this study identified DNA damage repair, one of the multiple functions of TERA/VCP/p97, as a new common pathomechanism of polyQ diseases, it remains unclear to which extent DNA damage repair and other functions of TERA/VCP/ p97 contribute to the polyQ disease pathology. Although our results suggested that contribution of TERA/VCP/p97-mediated ERAD was not large in neuronal pathology, it is not clear how our findings are correlated to autophagy. There are a number of evidences that activation of brain autophagy by chemicals attenuates toxicity of mutant $\mathrm{Htt}$ protein ${ }^{51-53}$ and that a certain defect of autophagy contributes to other neurodegenerative disorders like Alzheimer's disease $\mathrm{e}^{54}$ and Parkinson's disease ${ }^{55}$. Meanwhile, a report showed that an mTOR inhibitor was effective for the muscle pathology but not brain pathology of $\mathrm{HD}^{56}$, and another group recently reported that a new mouse model of SCA6 did not show any change of autophagy in the brain $^{57}$. In some preliminary studies, we also investigated macroautophagy in Purkinje cells of mutant Atxn1-KI mice by electron microscopy, but we have not been able to detect any changes.

Moreover, multiple pathways like transcription, including PGC-1alpha, splicing, mitochondrial stress, ER stress and oxidative stress mediated by other common molecules may underlie degeneration. Therefore, we need fundamentally new technologies to quantitatively evaluate contribution of each pathomechanism (or pathway) to the pathology and symptom, and solution of such issues would greatly advance our understanding of the common pathomechanism of neurodegeneration and would help developing radical therapeutics based on the common pathology.

\section{Methods}

Filter-binding assay. HeLa cells were transfected by different amounts of Htt$103 \mathrm{Q}$ expression vector $\left(0,0.25,0.5,1,2,4 \mu \mathrm{g} / 4 \times 10^{-4}\right.$ cells). After $36 \mathrm{~h}$, cells were harvested and lysed with PBS- $0.02 \%$ Nonidet P-40. The sup after centrifugation was filtrated by Durapore-PDVF $(0.22 \mu \mathrm{m})$. The flow-through fraction was blotted with anti-VCP antibody (BD Transduction Laboratories, 1:1000). The signal intensities were corrected by GAPDH.

ERAD analysis. HEK 293 cells were plated in 24-well plates and transfected with pCl-neo-HA-CD3 $\Delta$ (ERAD substrate) and polyQ disease protein expression vectors (HA-CD3 $\Delta 0.06 \mu \mathrm{g}$; FLAG-VCP, $0.9 \mu \mathrm{g}$; Myc-polyQ, 0.6-0.9 $\mu \mathrm{g}$; balanced by using a pCMV-3Tag-1A empty vector). After $24 \mathrm{~h}$, the cells were subjected to treatment with or without CHX (final concentration, $100 \mu \mathrm{g} \mathrm{ml}^{-1}$ ). Thirty-six hours after the transfection, the cells were harvested directly into $50 \mu$ l of SDS sample buffer and processed for SDS-PAGE and immunoblotting for CD3 $\Delta$. The relative amount of $\mathrm{HA}-\mathrm{CD} 3 \Delta$ was measured using western blotting and was normalized by the amount of tubulin, which served as the internal controls. Film images were taken by a scanner and processed using the Image J software package (http://rsb.info.nih.gov/ij/) to measure the band intensity.

For ERAD assay with cortical neurons from Htt-111Q-KI mice ${ }^{28}$ and ATXN1$154 \mathrm{Q}-\mathrm{KI}$ mice $^{29}$, primary neurons were prepared according to the method described previously ${ }^{37,47}$. The pCl-neo-HA-CD3 $\Delta$ was transfected into neurons by lipofectamine LTX with Plus reagent (Invitrogen). ERAD analysis was performed using $\mathrm{CHX}$ as described above.

ErbB2 analysis. Cells transfected with GFP-ErbB2 and polyQ proteins and/or VCP (12-well dishes, glass coverslips; GFP-ErbB2, $0.3 \mu \mathrm{g}$; FLAG-VCP, $0.3 \mu \mathrm{g}$; MycpolyQ, $0.4 \mu \mathrm{g}$ ) were incubated for $36 \mathrm{~h}$ and then fixed. The immunocytochemistry procedure mentioned above was performed to stain the Myc-polyQ and/or FLAGVCP proteins.

ATPase activity assay of VCP. ATPase activity of VCP was assayed following the method reported previously ${ }^{22}$. Fresh brains dissected control and Htt-111Q-KI mice were homogenized in $20 \mathrm{mM}$ HEPES ( $\mathrm{pH}=7.5$ ), $5 \mathrm{mM} \mathrm{MgCl} 2,2.5 \mathrm{mM}$ DTT, $150 \mathrm{mM} \mathrm{NaCl}$ by Dounce homogenizer, sonicated (10 $\mathrm{s} \times 3$ times), and centrifuged at $20,000 \mathrm{~g}$ for $10 \mathrm{~min}$. The sup was filtrated by $0.22 \mu \mathrm{m}$ PVDF membrane (Milipore) and protein concentration was measured by BCA Protein Assay (Thermo). The extracts $\left(5 \mathrm{mg} \mathrm{ml}^{-1}, 500 \mu \mathrm{l}\right)$ were applied to high performance liquid chromatography (HPLC, Shimadzu) and fractionated by gel filtration chromatography (Superdex 200 10/300 GL, GE Healthcare) and ion exchange chromatography (DEAE-2SW, Tosoh). Each fraction $(40 \mu \mathrm{l})$ was added to $50 \mu \mathrm{l}$ reaction buffer (0.2 mM ATP (SIGMA), $20 \mathrm{mM}$ HEPES $(\mathrm{pH}=7.5), 10 \mathrm{mM} \mathrm{MgCl}_{2}$ ) and incubated for $120 \mathrm{~min}$ at $37^{\circ} \mathrm{C}$. After additional incubation for $30 \mathrm{~min}$ at $37^{\circ} \mathrm{C}$ with BIOMOL GREEN (ENZO), phosphate ion released from ATP was assayed by measuring OD at $630 \mathrm{~nm}$ with plate reader (MULTISKAN ASCENT, Thermo). 
The values of phosphate ion were corrected with the amounts VCP in control and Htt-111Q-KI mice evaluated by western blot on a single membrane.

Laser microirradiation. Laser microirradiation and signal acquisition from damaged area were performed according to the method as described previously ${ }^{58}$. In the case of cell line, U2OS cells grown on $25-\mathrm{mm}$ coverslips were treated with $2 \mu \mathrm{M}$ Hoechst33258 (DOJINDO, Japan) for $20 \mathrm{~min}$ to sensitize the cells to DSBs. Using the software (AIM4.2, Carl Zeiss, Germany) of microscopy (LSM510META, Carl Zeiss, Germany), rectangle-shaped areas locating at cell nuclei were irradiated by UV laser (Maximum power: $30 \mathrm{~mW}$, laser output: $75 \%$, wavelength: $405 \mathrm{~nm}$, iteration: 5, pixel time: $12 \mu \mathrm{s}$ : zoom 6), and time-lapse images were obtained every $30 \mathrm{~s}$. In the case of neuron, primary mouse cortical neurons cultured on Lab-Tek II Chambered Coverglass System (Thermo Fischer Scientific Inc, MA, USA) with the medium described above were similarly irradiated expect using zoom at 2 , and signal acquisition was performed by immunocytochemistry with anti-VCP (Santa Cruz, H-120, 1:100) and anti- $\gamma \mathrm{H} 2 \mathrm{AX}$ (Millipore, JBW301, 1:1,000) antibodies at $1 \mathrm{~h}$ after irradiation. In both cases, region of interest matching exactly to the bleached area was determined using Adobe Photoshop CS3, and mean signal intensities per pixel of VCP protein were obtained from region of interest. Signal intensity was normalized by that of non-irradiated area, which was indicated as a normalized fluorescence unit. Lentiviral vectors were constructed by subcloning human full-length ataxin-1 cDNA or human huntingtin-exon1 cDNA from previously described plasmids ${ }^{37}$ into EcoRI-XbaI and XbaI-BamHI sites of pLVSIN-CMV Pur Vector (TaKaRa, Japan), respectively.

DSB repair complex formation. For interaction between VCP and L3MBTL1, HeLa cells $\left(2 \times 10^{6}\right)$ plated on $10 \mathrm{~cm}$ dishes were transfected with pCMV-FLAGVCP described above, and incubated for $36 \mathrm{~h}$. Cells were incubated for $1 \mathrm{~h}$ with $400 \mu \mathrm{g} \mathrm{ml}^{-1}$ of the radiomimetic drug Zeocin (Invitrogen) to induce DNA double strand breaks. After washing with PBS, cells were homogenized with $1 \mathrm{ml}$ of TNE buffer and rotated at $4{ }^{\circ} \mathrm{C}$ for $2 \mathrm{~h}$. Then, the solutions were centrifuged at $15,000 \mathrm{~g}$ for $15 \mathrm{~min}$. The supernatant were incubated with $20 \mu \mathrm{l}$ of protein G-sepharose beads (GE healthcare) for $2 \mathrm{~h}$ at $4{ }^{\circ} \mathrm{C}$ with rotation. After beads were removed, the lysates were incubated with $2.5 \mu \mathrm{g}$ of anti-L3MBTL1 antibody overnight at $4{ }^{\circ} \mathrm{C}$ with rotation, followed by the incubation with protein G-sepharose for $2 \mathrm{~h}$. The beads were washed five times with $500 \mu \mathrm{l}$ of TNE buffer and resolved using SDSPAGE sample buffer.

Characterization of cytoplasmic vacuoles. HeLa cells $\left(1 \times 10^{5}\right)$ were plated on glass coverslips in 12-well plates and transfected with a Myc-polyQ protein expression vector as described. Thirty-six hours after transfection, cells were fixed and stained with anti-Myc (Santa Cruz Biotechnology; 9E10) antibody, and the co-localization of Myc-polyQ with EGFP-LC3 (autophagosome marker) or DsRed containing an ER translocation sequence (KDEL) was examined. Cells possessing cytoplasmic vacuoles (classified by $10-\mu \mathrm{m}$ diameter) were counted in 50 visual fields.

Immunohistochemistry of polyQ disease model mice. Fresh brains of heterozygous Htt-111Q-KI (48 weeks) ${ }^{28}$, ATXN1-154Q-KI (32 weeks) ${ }^{29}$, Htt-Tg (R6/2) mice $^{30}$ (12 weeks), ATXN7-92Q-Tg mice (33 weeks) ${ }^{31}$ or AR-97Q-Tg mice ${ }^{32}$ (13 weeks) were fixed in phosphate-buffered $4 \%$ paraformaldehyde and embedded in paraffin. Sections (thickness, 5-10 $\mu \mathrm{m}$ ) were dewaxed in xylene and rehydrated using a descending ethanol series. Sections were boiled in $10 \mathrm{mM}$ citrate buffer $(\mathrm{pH}$ 6.0) in a microwave oven three times and kept at room temperature for $30 \mathrm{~min}$ after the final boiling. Incubation in PBS containing $1 \%$ bovine serum albumin and $0.01 \%(\mathrm{v} / \mathrm{v})$ Triton X-100 was performed for $30 \mathrm{~min}$ to block nonspecific binding. Samples were incubated overnight at $4{ }^{\circ} \mathrm{C}$ with a primary antibody, followed by incubation with a secondary antibody for $1 \mathrm{~h}$ at room temperature. After a 2-min DAPI treatment, the brains were mounted using Fluoromount and covered using coverslips. Cells were visualized using confocal microscopy (Zeiss LSM510).

Primary antibodies and dilution conditions were as follows: anti-Htt antibody (HD1, 1:100), anti-ATXN1 antibody (11NQ, mouse 1:100), and anti-VCP antibody (Cell Signalling Technologies; rabbit, 1:100). Secondary antibodies and dilution conditions were as follows: Alexa Fluor 488-conjugated anti-mouse antibody (Molecular Probes; 1:1000) and Cy3-conjugated anti-rabbit antibody (Jackson; $1: 1,000)$

Immunohistochemistry of human brain tissues. Brains of genotype-diagnosed patients with HD or SCA1 were fixed in phosphate-buffered $4 \%$ paraformaldehyde and embedded in paraffin. Sections (thickness, $10 \mu \mathrm{m}$ ) were dewaxed in xylene and rehydrated using a descending ethanol series. Treatment and observation of the sections were similar to the methods for mouse brain tissues except for the antibody conditions. Primary antibodies and dilution conditions were as follows: anti-Htt antibody (HD1, 1:200), anti-ATXN1 antibody (H21, goat 1:100), and anti-VCP antibody (Becton Dickinson and Company; 1:200). Secondary antibodies and dilution conditions were as follows: Alexa Fluor 488-conjugated anti-mouse antibody (Molecular Probes; 1:1,000) and Cy3-conjugated anti-rabbit antibody (Jackson; 1:1,000).
Ethics statement. This study was performed in strict accordance with the recommendations in the Guide for the Care and Use of Laboratory Animals of the National Institutes of Health, and had been approved by the Committees on Human Ethics and Animal Experiments of the Tokyo Medical and Dental University (Number: 2011-22-3 and 0130225).

\section{References}

1. Watts, G. D. et al. Inclusion body myopathy associated with Paget disease of bone and frontotemporal dementia is caused by mutant valosin-containing protein. Nat. Genet. 36, 377-381 (2004).

2. Johnson, J. O. et al. Exome sequencing reveals VCP mutations as a cause of familial ALS. Neuron 68, 857-864 (2010).

3. Imafuku, I. et al. Polar amino acid-rich sequences bind to polyglutamine tracts. Biochem. Biophys. Res. Commun. 253, 16-20 (1998).

4. Hirabayashi, M. et al. VCP/p97 in abnormal protein aggregates, cytoplasmic vacuoles, and cell death, phenotypes relevant to neurodegeneration. Cell Death Differ. 8, 977-984 (2001).

5. Woodman, P.G. p97, a protein coping with multiple identities. J. Cell Sci. 116, 4283-4290 (2003).

6. Zhang, L., Ashendel, C. L., Becker, G. W. \& Morré, D. J. Isolation and characterization of the principal ATPase associated with transitional endoplasmic reticulum of rat liver. J. Cell Biol. 127, 1871-1883 (1994).

7. Rabouille, C., Levine, T. P., Peters, J. M. \& Warren, G. An NSF-like ATPase, p97, and NSF mediate cisternal regrowth from mitotic Golgi fragments. Cell 82, 905-914 (1995).

8. Pleasure, I. T., Black, M. M. \& Keen, J. H. Valosin-containing protein, VCP, is a ubiquitous clathrin-binding protein. Nature 365, 459-462 (1993).

9. Ye, Y., Shibata, Y., Yun, C., Ron, D. \& Rapoport, T. A. A membrane protein complex mediates retro-translocation from the ER lumen into the cytosol. Nature 429, 841-847 (2004).

10. Ye, Y., Meyer, H. H. \& Rapoport, T. A. The AAA ATPase Cdc48/p97 and its partners transport proteins from the ER into the cytosol. Nature 414, 652-656 (2001).

11. Zhong, X. et al. AAA ATPase p97/valosin-containing protein interacts with gp78, a ubiquitin ligase for endoplasmic reticulum-associated degradation. J. Biol. Chem. 279, 45676-45684 (2004).

12. Kittler, R. et al. An endoribonuclease-prepared siRNA screen in human cells identifies genes essential for cell division. Nature 432, 1036-1040 (2004).

13. Acs, K. et al. The AAA-ATPase VCP/p97 promotes 53BP1 recruitment by removing L3MBTL1 from DNA double-strand breaks. Nat. Struct. Mol. Biol. 18, 1345-1350 (2011).

14. Meerang, M. et al. The ubiquitin-selective segregase $\mathrm{VCP} / \mathrm{p} 97$ orchestrates the response to DNA double-strand breaks. Nat. Cell. Biol. 13, 1376-1382 (2011).

15. Wójcik, C., Yano, M. \& DeMartino, G.N. RNA interference of valosincontaining protein $(\mathrm{VCP} / \mathrm{p} 97)$ reveals multiple cellular roles linked to ubiquitin/proteasome-dependent proteolysis. J. Cell Sci. 117, 281-292 (2004).

16. Dalal, S., Rosser, M.F., Cyr, D.M. \& Hanson, P.I. Distinct roles for the AAA ATPases NSF and p97 in the secretory pathway. Mol. Biol. Cell 15, 637-648 (2004).

17. Mimnaugh, E. G., Xu, W., Vos, M., Yuan, X. \& Neckers, L. Endoplasmic reticulum vacuolization and valosin-containing protein relocalization result from simultaneous hsp90 inhibition by geldanamycin and proteasome inhibition by velcade. Mol. Cancer Res. 4, 667-681 (2006).

18. Ju, J.S. et al. Valosin-containing protein (VCP) is required for autophagy and is disrupted in VCP disease. J. Cell Biol. 187, 875-888 (2009).

19. Weihl, C.C., Miller, S.E., Hanson, P.I. \& Pestronk, A. Transgenic expression of inclusion body myopathy associated mutant p97/VCP causes weakness and ubiquitinated protein inclusions in mice. Hum. Mol. Genet. 16, 919-928 (2007).

20. Custer, S.K., Neumann, M., Lu, H., Wright, A.C. \& Taylor, J.P. Transgenic mice expressing mutant forms $\mathrm{VCP} / \mathrm{p} 97$ recapitulate the full spectrum of IBMPFD including degeneration in muscle, brain and bone. Hum. Mol. Genet. 19, 1741-1755 (2010).

21. Badadani, M. et al. VCP associated inclusion body myopathy and paget disease of bone knock-in mouse model exhibits tissue pathology typical of human disease. PLoS One 5, e13183 (2010).

22. Halawani, D. et al. Hereditary inclusion body myopathy-linked p97/VCP mutations in the $\mathrm{NH} 2$ domain and the D1 ring modulate p97/VCP ATPase activity and D2 ring conformation. Mol. Cell Biol. 29, 4484-4494 (2009).

23. Manno, A., Noguchi, M., Fukushi, J., Motohashi, Y. \& Kakizuka, A. Enhanced ATPase activities as a primary defect of mutant valosin-containing proteins that cause inclusion body myopathy associated with Paget disease of bone and frontotemporal dementia. Genes Cells 15, 911-922 (2010).

24. Koike, M. et al. Valosin-containing protein (VCP) in novel feedback machinery between abnormal protein accumulation and transcriptional suppression. J. Biol. Chem. 285, 21736-21749 (2010). 
25. Chai, Y., Shao, J., Miller, V. M., Williams, A. \& Paulson, H. L. Live-cell imaging reveals divergent intracellular dynamics of polyglutamine disease proteins and supports a sequestration model of pathogenesis. Proc. Natl Acad. Sci. USA 99, 9310-9315 (2002)

26. Stenoien, D. L., Mielke, M. \& Mancini, M. A. Intranuclear ataxin1 inclusions contain both fast- and slow-exchanging components. Nat. Cell Biol. 4, 806-810 (2002).

27. Kim, S., Nollen, E. A., Kitagawa, K., Bindokas, V. P. \& Morimoto, R. I. Polyglutamine protein aggregates are dynamic. Nat. Cell Biol. 4, 826-831 (2002).

28. Wheeler, V. C. et al. Length-dependent gametic CAG repeat instability in the Huntington's disease knock-in mouse. Hum. Mol. Genet. 8, 115-122 (1999).

29. Watase, K. et al. A long CAG repeat in the mouse Scal locus replicates SCA1 features and reveals the impact of protein solubility on selective neurodegeneration. Neuron 34, 905-919 (2002).

30. Mangiarini, L. et al. Exon 1 of the HD gene with an expanded CAG repeat is sufficient to cause a progressive neurological phenotype in transgenic mice. Cell 87, 493-506 (1996).

31. La Spada, A. R. et al. Polyglutamine-expanded ataxin-7 antagonizes CRX function and induces cone-rod dystrophy in a mouse model of SCA7. Neuron 31, 913-927 (2001)

32. Katsuno, M. et al. Testosterone reduction prevents phenotypic expression in a transgenic mouse model of spinal and bulbar muscular atrophy. Neuron 35 , 843-854 (2002).

33. Fry, W.H., Simion, C., Sweeney, C. \& Carraway, K. L. Quantity control of the ErbB3 receptor tyrosine kinase at the endoplasmic reticulum. Mol. Cell Biol. 31, 3009-3018 (2011).

34. Ju, J. S. \& Weihl, C. C. Inclusion body myopathy, Paget's disease of the bone and fronto-temporal dementia: a disorder of autophagy. Hum. Mol. Genet. 19, R38-R45 (2010).

35. Tamura, T. et al. Ku70 alleviates neurodegeneration in Drosophila models of Huntington's disease. PLoS One 6, e27408 (2011).

36. Qi, M. L. et al. Proteome analysis of soluble nuclear proteins reveals that HMGB1/2 suppress genotoxic stress in polyglutamine diseases. Nat. Cell Biol. 9, 402-414 (2007).

37. Enokido, Y. et al. Mutant huntingtin impairs Ku70-mediated DNA repair. J. Cell Biol. 189, 425-443 (2010).

38. $\mathrm{Hu}$, Y. et al. Transcriptional modulator $\mathrm{H} 2 \mathrm{~A}$ histone family, member $\mathrm{Y}$ (H2AFY) marks Huntington disease activity in man and mouse. Proc. Natl Acad. Sci. USA 108, 17141-17146 (2011).

39. Ehrlich, M. E. \& Gandy, S. Chromatin plasticity and the pathogenesis of Huntington disease. Proc. Natl Acad. Sci. USA 108, 16867-16868 (2011)

40. Ramadan, K. et al. Cdc48/p97 promotes reformation of the nucleus by extracting the kinase Aurora B from chromatin. Nature 450, 1258-1262 (2007).

41. McShea, A., Harris, P.L., Webster, K.R., Wahl, A.F. \& Smith, M.A. Abnormal expression of the cell cycle regulators P16 and CDK4 in Alzheimer's disease. Am. J. Pathol. 150, 1933-1939 (1997).

42. Busser, J., Geldmacher, D.S. \& Herrup, K. Ectopic cell cycle proteins predict the sites of neuronal cell death in Alzheimer's disease brain. J. Neurosci. 18, 28012807 (1998).

43. Kruman, I.I. et al. Cell cycle activation linked to neuronal cell death initiated by DNA damage. Neuron 41, 549-561 (2004).

44. Al-Ramahi, I. et al. dAtaxin-2 mediates expanded Ataxin-1-induced neurodegeneration in a Drosophila model of SCA1. PLoS Genet. 3, e234 (2007).

45. Stelzl, U. et al. A human protein-protein interaction network: a resource for annotating the proteome. Cell 122, 957-968 (2005).

46. Lim, J. et al. A protein-protein interaction network for human inherited ataxias and disorders of Purkinje cell degeneration. Cell 125, 801-814 (2006).

47. Kahle, J.J. et al. Comparison of an expanded ataxia interactome with patient medical records reveals a relationship between macular degeneration and ataxia. Hum. Mol. Genet. 20, 510-527 (2011).

48. Guinto, J.B., Ritson, G.P., Taylor, J.P. \& Forman, M.S. Valosin-containing protein and the pathogenesis of frontotemporal dementia associated with inclusion body myopathy. Acta. Neuropathol. 114, 55-61 (2007).

49. Arrasate, M., Mitra, S., Schweitzer, E.S., Segal, M.R. \& Finkbeiner, S. Inclusion body formation reduces levels of mutant huntingtin and the risk of neuronal death. Nature 431, 805-810 (2004).
50. Tagawa, K. et al. Distinct aggregation and cell death patterns among different types of primary neurons induced by mutant huntingtin protein. J. Neurochem. 89, 974-987 (2004).

51. Ravikumar, B. et al. Inhibition of mTOR induces autophagy and reduces toxicity of polyglutamine expansions in fly and mouse models of Huntington disease. Nat. Genet. 36, 585-595 (2004).

52. Rose, C. et al. Rilmenidine attenuates toxicity of polyglutamine expansions in a mouse model of Huntington's disease. Hum. Mol. Genet. 19, 2144-2153 (2010).

53. Tsvetkov, AS. et al. A small-molecule scaffold induces autophagy in primary neurons and protects against toxicity in a Huntington disease model. Proc Natl Acad. Sci. USA 107, 16982-16987 (2010).

54. Boland, B. et al. Autophagy induction and autophagosome clearance in neurons: relationship to autophagic pathology in Alzheimer's disease. J. Neurosci. 28, 6926-6937 (2008).

55. Crews, L. et al. Selective molecular alterations in the autophagy pathway in patients with Lewy body disease and in models of alpha-synucleinopathy. PLoS One 5, e9313 (2010).

56. Fox, JH. et al. The mTOR kinase inhibitor Everolimus decreases S6 kinase phosphorylation but fails to reduce mutant huntingtin levels in brain and is not neuroprotective in the R6/2 mouse model of Huntington's disease. Mol. Neurodegener. 5, 26 (2010).

57. Unno, T. et al. Development of Purkinje cell degeneration in a knockin mouse model reveals lysosomal involvement in the pathogenesis of SCA6. Proc Natl Acad. Sci. USA 109, 17693-17698 (2012).

58. Lukas, C, Falck, J, Bartkova, J, Bartek, J \& Lukas, J. Distinct spatiotemporal dynamics of mammalian checkpoint regulators induced by DNA damage. Nat. Cell. Biol. 5, 255-260 (2003).

\section{Acknowledgements}

This work was supported by a Grant-in-aid for Scientific Research on Innovative Areas (Foundation of Synapse and Neurocircuit Pathology) and Strategic Research Program for Brain Sciences (SRPBS) from the Ministry of Education, Culture, Sports, Science and Technology of Japan; CREST from Japan Science Technology Agency; a Grant-in-aid from the Research Committee for Ataxic Disease from the Ministry of Health, Labour and Welfare to HO; and R01s NS41648 and AG33082 to ARL. We thank Prof. Huda Y. Zoghbi (Baylor College of Medicine) and Dr Kei Watase (TMDU) for the ATXN1-KI mice, Professor Marcy MacDonald (Harvard Medical School) for the Htt-KI mice, Professor Tom A. Rapoport (Harvard Medical School) for the Derlin-1 antibody and technical advice, Professor Diane Merry (Thomas Jefferson University) for the AR plasmids, and Professor Len Neckers (National Institute of Health) for the ErbB2 plasmid, Professor Randall Pittman (University of Pennsylvania) for the pCI-neo-HA-CD3d plasmid, and Prof. Tamotsu Yoshitomi (National Institute for Basic Biology) and Prof. Noboru Mizushima (Tokyo Medical and Dental University) for the pEGFP-LC3. We thank Dr Kei Watase (Tokyo Medical and Dental University) for technical support and critical discussion. We also thank Ms Chie IInuma, Ms Chiharu Mizoi, Ms Aiko Unno, Ms Chiharu Nagashima and Ms Tayoko Tajima for technical support.

\section{Author contributions}

K.F., Y.N., T.O., and H.I. designed and performed experiments, analysed data and wrote the paper. T.T., K.T., T.S., A.K., K.M., H.S., M.S. and C.Y. performed a part of experiments and dada analysis. Y.E. and M.M. collected human brains and prepared samples. M.K. and G.S generated mouse model and prepared samples. K.K. and S.T. performed a part of experiments. J.P.T. and A.R.LS wrote the paper. H.O. designed all experiments and wrote the paper.

\section{Additional information}

Supplementary Information accompanies this paper at http://www.nature.com/ naturecommunications

Competing financial interests: The authors declare no competing financial interests.

Reprints and permission information is available online at http://npg.nature.com/ reprintsandpermissions/

How to cite this article: Fujita, K. et al. A functional deficiency of TERA/VCP/p97 contributes to impaired DNA repair in multiple polyglutamine diseases. Nat. Commun. 4:1816 doi: $10.1038 /$ ncomms2828 (2013). 